\title{
THE INTERMEDIATE DISORDER REGIME FOR DIRECTED POLYMERS IN DIMENSION $1+1^{1}$
}

\author{
By Tom Alberts, Konstantin Khanin And JeRemy QuAstel
}

\author{
California Institute of Technology, University of Toronto \\ and University of Toronto
}

We introduce a new disorder regime for directed polymers in dimension $1+1$ that sits between the weak and strong disorder regimes. We call it the intermediate disorder regime. It is accessed by scaling the inverse temperature parameter $\beta$ to zero as the polymer length $n$ tends to infinity. The natural choice of scaling is $\beta_{n}:=\beta n^{-1 / 4}$. We show that the polymer measure under this scaling has previously unseen behavior. While the fluctuation exponents of the polymer endpoint and the log partition function are identical to those for simple random walk $(\zeta=1 / 2, \chi=0)$, the fluctuations themselves are different. These fluctuations are still influenced by the random environment, and there is no self-averaging of the polymer measure. In particular, the random distribution of the polymer endpoint converges in law (under a diffusive scaling of space) to a random absolutely continuous measure on the real line. The randomness of the measure is inherited from a stationary process $A_{\beta}$ that has the recently discovered crossover distributions as its one-point marginals, which for large $\beta$ become the GUE Tracy-Widom distribution. We also prove existence of a limiting law for the four-parameter field of polymer transition probabilities that can be described by the stochastic heat equation.

In particular, in this weak noise limit, we obtain the convergence of the point-to-point free energy fluctuations to the GUE Tracy-Widom distribution. We emphasize that the scaling behaviour obtained is universal and does not depend on the law of the disorder.

1. Introduction. The problem of directed polymers in a random environment was first studied in [26] and received its first mathematical treatment in [27]. Since then it has received considerable attention in the statistical physics and probability communities; see $[16,25]$ for reviews. In the setting of the $d$-dimensional integer lattice, the polymer measure is a random probability measure on paths of $d$ dimensional nearest neighbour lattice walks. The randomness of the polymer measure is inherited from an i.i.d. collection of random variables placed on the sites of $\mathbb{Z}_{+} \times \mathbb{Z}^{d}$. Collectively these variables are called the random environment. Given a fixed environment $\omega: \mathbb{Z}_{+} \times \mathbb{Z}^{d} \rightarrow \mathbb{R}$, the energy of an $n$-step nearest neighbour

Received April 2012; revised February 2013.

${ }^{1}$ Supported by the Natural Sciences and Engineering Research Council of Canada.

MSC2010 subject classifications. 60F05, 82C05.

Key words and phrases. Directed polymers, near-critical scaling limits, $U$-statistics, KPZ equation. 
walk $S$ is

$$
H_{n}^{\omega}(S)=\sum_{i=1}^{n} \omega\left(i, S_{i}\right) .
$$

The polymer measure on such walks is then defined in the usual Gibbsian way by

$$
\mathbf{P}_{n, \beta}^{\omega}(S)=\frac{1}{Z_{n}^{\omega}(\beta)} e^{\beta H_{n}^{\omega}(S)} \mathbf{P}(S),
$$

where $\beta>0$ is the inverse temperature, $\mathbf{P}$ is the symmetric simple random walk measure on paths started at the origin and $Z_{n}^{\omega}(\beta)$ is the partition function

$$
Z_{n}^{\omega}(\beta)=\mathbf{P}\left[e^{\beta H_{n}^{\omega}(S)}\right] .
$$

The random environment is a probability measure $Q$ on the space of environments $\Omega=\left\{\omega: \mathbb{Z}_{+} \times \mathbb{Z}^{d} \rightarrow \mathbb{R}\right\}$. We let $Q$ be a product measure so that the variables $\omega(i, z)$ are independent and identically distributed, and we make the assumption that the $\omega$ have moments of all orders and that

$$
\lambda(\beta):=\log Q e^{\beta \omega}<\infty,
$$

at least for $\beta$ sufficiently small. In the conclusion, we will describe work in progress for relaxing this assumption. Note that throughout we use $\mathbf{P}$ and $Q$ to denote expectation as well as probability.

The overall goal of the subject is to study the behavior of the polymer as $\beta$ and $d$ vary and $n$ gets large. At $\beta=0$ the polymer measure is the simple random walk; hence the walk is entropy dominated and exhibits diffusive behavior. For $\beta$ large the polymer measure concentrates on paths with high energy and the diffusive behavior is no longer guaranteed. Entropy domination of the measure is called weak disorder, and energy domination is called strong disorder. The precise separation between these two regimes is defined in terms of the positivity of the limit of the martingale $e^{-n \lambda(\beta)} Z_{n}^{\omega}(\beta)$, as $n \rightarrow \infty$. The weak disorder regime consists of $\beta$ for which

$$
\lim _{n \rightarrow \infty} e^{-n \lambda(\beta)} Z_{n}^{\omega}(\beta)>0,
$$

whereas if the limit is zero, then $\beta$ is said to be in the strong disorder regime. For $d \geq 3$, it was shown early on $[10,27]$ that weak disorder holds for small $\beta$. Later, Comets and Yoshida [19] showed that in every dimension there is a critical value $\beta_{c}$ such that weak disorder holds for $0 \leq \beta<\beta_{c}$ and strong disorder for $\beta>\beta_{c}$. In addition, for $d=1$ and 2 they prove that $\beta_{c}=0$. In the rest of this paper we focus exclusively on the case $d=1$ so that all positive $\beta$ are in the strong disorder regime.

Understanding the polymer behavior in the strong disorder regime is one of our main goals. Strong disorder manifests itself in a variety of ways that have become 
more evident in recent years. Arguably the most well-known phenomenon is superdiffusivity of the paths under the polymer measure. This is usually expressed through an exponent $\zeta$, and although there is no commonly agreed upon definition of $\zeta$ in the literature, it is roughly meant to be the exponent such that

$$
\left|S_{n}\right| \sim n^{\zeta}
$$

as $n \rightarrow \infty$, for "typical" realizations of $\omega$. For $d=1$ the long-standing conjecture is $\zeta=2 / 3$, but at present has been obtained only in models with specific weights and sometimes boundary conditions. The best result is by Seppäläinen [39] for a model with specific weights and boundary conditions. There are also upper and lower bounds (neither one sharp) given for certain special models [18, 31, 34, 45, 46]. The picture is very different from that of simple random walk where $\zeta=1 / 2$, and the polymer endpoint is roughly uniformly distributed on intervals of length $\sqrt{n}$. For positive $\beta$ the polymer is localized and most of the endpoint density sits in a relatively small region around a random point at distance $n^{2 / 3}$ from the origin. The size of this region is of much smaller order than $n^{2 / 3}$. In fact, it is believed that the variance of the polymer endpoint is order one. Carmona and $\mathrm{Hu}[13]$ and Comets et al. [15] showed that there is a constant $c_{0}=c_{0}(\beta)>0$ such that the event

$$
\limsup _{n \rightarrow \infty} \max _{x \in \mathbb{Z}} \mathbf{P}_{n, \beta}^{\omega}\left(S_{n}=x\right) \geq c_{0}
$$

has $Q$ probability one. This phenomenon is called strong localization. It is in stark contrast to the simple random walk case where the supremum decays like $n^{-1 / 2}$.

Strong disorder also has an effect on the large time behavior of the partition function. First, for $\beta>0$, there is the well-known inequality

$$
\begin{aligned}
\rho(\beta) & :=\lim _{n \rightarrow \infty} \frac{\log Z_{n}^{\omega}(\beta)}{n}=\lim _{n \rightarrow \infty} \frac{Q \log Z_{n}^{\omega}(\beta)}{n}<\lim _{n \rightarrow \infty} \frac{\log Q Z_{n}^{\omega}(\beta)}{n} \\
& =\lambda(\beta)
\end{aligned}
$$

between the quenched and annealed free energies (the second equality is by a subadditivity argument and some concentration estimates; see, for example, $[13,15])$. The inequality is partially the standard annealing bound, but the fact that it is strict is a feature of strong disorder that was proved in $d=1$ by Comets et al. [17]. Quantitative bounds on the size of the gap were later proved in [30]. When strict inequality holds, $\beta$ is said to be in the very strong disorder regime, and hence in $d=1$ very strong disorder and strong disorder are equivalent. From (1) the leading term behavior of the $\log$ of the partition function is $\rho(\beta) n$, and the randomness is conjectured to appear through a lower order term

$$
\log Z_{n}^{\omega}(\beta)=\rho(\beta) n+c(\beta) n^{\chi} X
$$

The fluctuation exponent $\chi$ is believed to be $1 / 3$ for $d=1$, and the random fluctuations $X$ are expected to converge to the Tracy-Widom GOE distribution [40] 
arising from the analogous asymptotics for the largest eigenvalue from the Gaussian orthogonal ensemble. Observe that the conjectured values of $\zeta$ and $\chi$ satisfy the simple relation (KPZ relation)

$$
\chi=2 \zeta-1
$$

Versions of this were recently proved rigorously in [14] and [5], under the assumption that the exponents exist as appropriate limits. In the $\beta=\infty$ case of last passage percolation Johannson has established, for a particular distribution for the environment variables, that the scaling exponents $\chi=1 / 3$ and $\zeta=2 / 3$ are correct and that the fluctuations are of Tracy-Widom type, but rigorous mathematical proofs remain elusive in the $\beta<\infty$ case of directed polymers. Seppäläinen [39] managed to give proofs of the exponents for the log-gamma model with a specific choice of the environment and some boundary conditions, but the more general case remains open.

As the description above indicates, much of the conceptual picture for polymers in the strong disorder regime is understood, but little of it is rigorously proved. In this paper we introduce a new disorder regime that is interesting in its own right, and for which we are also able to prove many results. We call this regime the intermediate disorder regime, and it is exclusive to the $d=1$ case. It corresponds to a scaling of the inverse temperature with the length of the polymer. The name is chosen because it sits between weak and strong disorder and features of both are present. While the fluctuation exponents for intermediate disorder coincide with those for weak disorder $(\zeta=1 / 2, \chi=0)$, the fluctuations themselves, as in the strong disorder regime, are not decoupled from the random environment. In particular, in contrast to the weak disorder case, the polymer measure does not converge to a single deterministic limit. Instead, under the diffusive scalings, the law of the random polymer measure converges to a limiting universal law, universal in the sense that it does not depend on the particular distribution of the environment. In the $1+1$-dimensional case we will prove:

Under the scaling $\beta_{n}=\beta n^{-1 / 4}$ the following is true:

- The partition function fluctuation exponent $\chi$ is 0 , and the partition function

$$
e^{-n \lambda\left(\beta n^{-1 / 4}\right)} Z_{n}^{\omega}\left(\beta n^{-1 / 4}\right)
$$

converges in law to a nondegenerate random variable $\mathcal{Z}_{\sqrt{2} \beta}$. The limiting random variable is square integrable, and its Wiener chaos decomposition is explicit; see (7).

- The path fluctuation exponent $\zeta$ is $1 / 2$, and the law of the distribution of $S_{n} / \sqrt{n}$ converges to a random density on the real line. In particular this implies the absence of localization. More precisely, there is a random local limit theorem for the endpoint density

$$
\left\{x \mapsto \frac{\sqrt{n}}{2} \mathbf{P}_{n, \beta_{n}}^{\omega}\left(S_{n}=x \sqrt{n}\right)\right\} \stackrel{(d)}{\longrightarrow}\left\{x \mapsto \frac{1}{\mathcal{Z}_{\sqrt{2} \beta}} e^{A_{\sqrt{2} \beta}(x)} e^{-x^{2} / 2} d x\right\},
$$


where $x \mapsto A_{\beta}(x)$ is a one-parameter family of stationary processes whose onepoint marginal distributions $G_{\beta}$ are the so-called crossover distributions, introduced in $[4,37]$.

- Under the intermediate disorder scaling and a diffusive scaling of space and time, the polymer transition probabilities converge in law as $n \rightarrow \infty$, that is,

$$
\begin{gathered}
\left\{(s, y ; t, x) \mapsto \frac{\sqrt{n}}{2} \mathbf{P}_{n, \beta_{n}}^{\omega}\left(S_{n t}=x \sqrt{n} \mid S_{n s}=y \sqrt{n}\right)\right\} \\
\stackrel{(d)}{\longrightarrow} \frac{\mathcal{Z}_{\sqrt{2} \beta}(s, y ; t, x) \int \mathcal{Z}_{\sqrt{2} \beta}(t, x ; 1, \lambda) d \lambda}{\mathcal{Z}_{\sqrt{2} \beta}}
\end{gathered}
$$

for $0 \leq s<t \leq 1$ and $x, y \in \mathbb{R}$. Here $\mathcal{Z}_{\beta}(s, y ; t, x)$ is a random field determined by solutions to the stochastic heat equation with multiplicative noise, and again it has an explicit Wiener chaos expansion.

As $\beta$ varies, it is believed that the stationary processes $A_{\beta}(x)$ interpolate between a Gaussian process as $\beta \rightarrow 0$ and the Airy 2 process as $\beta \rightarrow \infty$. Convergence to Airy ${ }_{2}$ is currently only known on the level of the one-point marginal distributions, which were shown in $[4,37]$ to converge to Tracy-Widom GUE. This interpolation property justifies the name crossover and emphasizes the importance of the process $A_{\beta}(x)$.

The rest of this paper is organized as follows. In the next section we give a precise formulation of our main results and sketch the main ideas behind the proofs. In Section 3 we provide some background material on white noise and stochastic integration, and in Section 4 we develop the theory of $U$-statistics on a spacetime domain. These theorems form the main technical component of our paper. In Section 5 we use the $U$-statistics results to prove Theorem 2.1. The proofs of Theorems 2.2 and 2.7 follow in Sections 6.1 and 6.2, although they are very similar to what is done in Section 5. The proofs of the tightness for Theorems 2.2 and 2.7 are based on standard SPDE arguments adapted to our situation, and we defer them until the Appendix. We end the main text in Section 7 with some remarks and ideas for future work.

2. Formulation of main results. We begin this section with a brief explanation of why the $n^{-1 / 4}$ scaling is the appropriate one, and then proceed with precise formulations of the results and some ideas of the proofs.

2.1. Critical scaling. It is not immediately obvious why $n^{-1 / 4}$ should be the critical scaling for intermediate disorder, and there is more than one heuristic explanation that can be given; see [12], for example. The simplest one is in terms of the partition function. Under the $n^{-1 / 4}$ scaling it has the form

$$
Z_{n}^{\omega}\left(\beta n^{-1 / 4}\right)=\mathbf{P}\left[\exp \left\{\beta n^{-1 / 4} H_{n}^{\omega}(S)\right\}\right] .
$$


Expanding the exponential as a Taylor series and keeping only the terms up to order $n^{-1 / 4}$ gives

$Z_{n}^{\omega}\left(\beta n^{-1 / 4}\right) \approx \mathbf{P}\left[1+\beta n^{-1 / 4} \sum_{i=1}^{n} \omega\left(i, S_{i}\right)\right]=1+\beta n^{-1 / 4} \sum_{i=1}^{n} \sum_{x \in \mathbb{Z}} \omega(i, x) \mathbf{P}\left(S_{i}=x\right)$.

By computing the variance of the right-hand side it is easily checked that the $n^{-1 / 4}$ scaling keeps the random term bounded. In fact, as the $\omega(i, x)$ variables are i.i.d. with mean zero and variance one, it is a simple exercise with characteristic functions to show that

$$
\beta n^{-1 / 4} \sum_{i=1}^{n} \sum_{x \in \mathbb{Z}} \omega(i, x) \mathbf{P}\left(S_{i}=x\right) \stackrel{(d)}{\longrightarrow} N\left(0, \sigma^{2}\right),
$$

where $\sigma^{2}=2 \beta^{2} / \sqrt{\pi}$. Hence, up to first-order at least, the partition function converges in law under the $n^{-1 / 4}$ scaling. In fact the same is also true of the higherorder terms. One can simply expand the exponential into a full power series, switch the expectation on paths with the summation and then analyze each term individually. For technical reasons, however, it is much easier to make the $e^{x} \approx 1+x$ approximation first and consider instead the slightly modified partition function

$$
\mathfrak{Z}_{n}^{\omega}\left(\beta n^{-1 / 4}\right)=\mathbf{P}\left[\prod_{i=1}^{n}\left(1+\beta n^{-1 / 4} \omega\left(i, S_{i}\right)\right)\right] .
$$

This partition function is the one that was originally introduced and studied (without any scaling) in the seminal papers $[10,27]$ for random \pm 1 environment variables. The advantage of the $\mathfrak{Z}_{n}^{\omega}$ partition function is that it can be more easily analyzed by expanding the product along each path, leading to

$$
\mathfrak{Z}_{n}^{\omega}\left(\beta n^{-1 / 4}\right)=\mathbf{P}\left[1+\sum_{k=1}^{n} \beta^{k} n^{-k / 4} \sum_{\mathbf{i} \in D_{k}^{n}} \prod_{j=1}^{k} \omega\left(\mathbf{i}_{j}, S_{\mathbf{i}_{j}}\right)\right]
$$

Here $D_{k}^{n}$ is the discrete integer simplex

$$
D_{k}^{n}=\left\{\mathbf{i}=\left(i_{1}, \ldots, i_{k}\right) \in \mathbb{N}^{k}: 1 \leq i_{1}<\cdots<i_{k} \leq n\right\} .
$$

For each $\mathbf{i} \in D_{k}^{n}$ we now average over the possible configurations of the random walk path at those times. By the Markov property for simple random walk, the probability of each configuration is given by the usual product of the transition kernels. Hence

$$
\begin{aligned}
& \mathfrak{Z}_{n}^{\omega}\left(\beta n^{-1 / 4}\right) \\
& \quad=1+\sum_{k=1}^{n} \beta^{k} n^{-k / 4} \sum_{\mathbf{i} \in D_{k}^{n}} \sum_{\mathbf{x} \in \mathbb{Z}^{k}} \prod_{j=1}^{k} \omega\left(\mathbf{i}_{j}, \mathbf{x}_{j}\right) p\left(\mathbf{i}_{j}-\mathbf{i}_{j-1}, \mathbf{x}_{j}-\mathbf{x}_{j-1}\right)
\end{aligned}
$$


where $\mathbf{i}_{0}=\mathbf{x}_{0}=0$, and $p(i, x)=\mathbf{P}\left(S_{i}=x\right)$ is the simple random walk transition kernel. This is the full expansion of $\mathfrak{Z}_{n}^{\omega}\left(\beta n^{-1 / 4}\right)$ into terms of all orders, and it is possible to analyze each order individually. The $k=1$ term we have already shown converges to a normal random variable. Unfortunately it is not as easy to write down the limiting distribution of the individual $k>1$ terms of the summation, but an explicit form of the limiting variables can easily be guessed. Very roughly speaking, if one scales space and time diffusively then the random walk transition probabilities in (6) approach the transition probabilities for a Brownian motion, and the environment variables $\omega$ on $\mathbb{Z}_{+} \times \mathbb{Z}$ begin to look a white noise on $\mathbb{R}_{+} \times \mathbb{R}$. The sums then become multiple integrals (over free space and ordered time) of the white noise weighted by the transition kernels for Brownian motion. This type of multiple stochastic integral is the $k$ th order term of a Wiener chaos expansion.

2.2. Main results and ideas of the proof. In this section we formulate our main results and provide sketches of the proofs. We believe that these sketches provide sufficient insight for many readers, and the formal proofs can be found beginning in Section 4. The power series expansion of the previous section brings us to the following theorem:

THEOREM 2.1. We have the following:

- Assume that the $\omega$ variables have mean zero and variance one. Then as $n \rightarrow \infty$,

$$
\mathfrak{Z}_{n}^{\omega}\left(\beta n^{-1 / 4}\right) \stackrel{(d)}{\longrightarrow} \mathcal{Z}_{\sqrt{2} \beta} .
$$

- Assuming that $\lambda(\beta)<\infty$ for $\beta$ small (with or without the normalizations on the mean and variance), we have the convergence

$$
e^{-n \lambda\left(\beta n^{-1 / 4}\right)} Z_{n}^{\omega}\left(\beta n^{-1 / 4}\right) \stackrel{(d)}{\longrightarrow} \mathcal{Z}_{\sqrt{2} \beta} .
$$

- The limiting variable $\mathcal{Z}_{\sqrt{2} \beta}$ can be identified as the sum of multiple stochastic integrals given by

(7) $\mathcal{Z}_{\sqrt{2} \beta}:=1+\sum_{k=1}^{\infty}(\sqrt{2} \beta)^{k} \int_{\Delta_{k}} \int_{\mathbb{R}^{k}} \prod_{i=1}^{k} W\left(t_{i}, x_{i}\right) \varrho\left(t_{i}-t_{i-1}, x_{i}-x_{i-1}\right) d x_{i} d t_{i}$.

Here $W(t, x)$ is a white noise on $\mathbb{R}_{+} \times \mathbb{R}$ with covariance $\mathrm{E}[W(t, x) W(s, y)]=$ $\delta(t-s) \delta(x-y), \Delta_{k}=\left\{0=t_{0}<t_{1}<t_{2}<\cdots<t_{k} \leq 1\right\}$ is the $k$-dimensional simplex, $x_{i} \in \mathbb{R}$ with $x_{0}=0$, and $\varrho$ is the standard Gaussian heat kernel

$$
\varrho(t, x)=\frac{e^{-x^{2} / 2 t}}{\sqrt{2 \pi t}} .
$$

REMARK. Note that the convergence of $\mathfrak{Z}_{n}^{\omega}$ only requires two moments for the environment variables; higher moments are not needed. The requirement that the variables have mean zero and variance one is only a normalization condition. 
REMARK. For the second statement on convergence of $Z_{n}^{\omega}$ we assumed that the $\omega$ have exponential moments, but we do not believe that this is necessary. In the conclusions we discuss our conjecture that six moments are sufficient for the convergence to go through.

REMARK. This theorem is in contrast to what is expected for the strong disorder case. Observe that

$$
\log Z_{n}^{\omega}\left(\beta n^{-1 / 4}\right)-n \lambda\left(\beta n^{-1 / 4}\right)
$$

converges (in law) as $n \rightarrow \infty$; hence it is an immediate corollary that $\chi=0$ under intermediate disorder.

Readers familiar with Gaussian Hilbert spaces will immediately recognize (7) as a Wiener chaos expansion. A good source for background material on Gaussian Hilbert spaces and Wiener chaos, and one that we will draw on throughout this work, is [28]. We include a brief background in Section 2. The distribution of this particular Wiener chaos (7) series is not known, though there are some intriguing conjectures [11]. Nonetheless it is still a very concrete expression to manipulate and study. Using the concept of Wick products (see [28]), we can rewrite (7) as

$$
\mathcal{Z}_{\sqrt{2} \beta}=\mathrm{E}_{0}\left[: \exp :\left\{\sqrt{2} \beta \int_{0}^{1} W\left(s, B_{S}\right) d s\right\}\right] .
$$

The expectation $\mathrm{E}_{0}$ is over 1-dimensional Brownian paths started at the origin. This shorthand is mostly formal since the integral of white noise over a Brownian path is not defined on a path-by-path basis. The procedure that is really indicated by (8) is to expand the exponential in a power series and then switch the expectation over paths with the summation of the series. Formally this produces the same series as in (7), except that one uses the Wick exponential : exp : as a reminder that powers of integrals should be expanded via the rule

$$
:\left(\int_{0}^{1} W\left(s, B_{s}\right) d s\right)^{k}:=k ! \int_{\Delta_{k}} \prod_{j=1}^{k} W\left(t_{j}, B_{t_{j}}\right) d t_{j}
$$

(recall $\Delta_{k}$ is the $k$-dimensional simplex). With this in mind it is easily checked that (7) and (8) are the same, and (8) should be viewed simply as shorthand for the well-defined Wiener chaos (7). The $\sqrt{2}$ factor in the exponential is a (rather annoying) feature of the periodicity of simple random walk. It can be seen as a manifestation of the factor of two in the local limit theorem

$$
p(n, x)=\frac{2}{\sqrt{2 \pi n}} e^{-x^{2} /(2 n)}+O\left(n^{-3 / 2}\right)=\frac{2}{\sqrt{n}} \varrho\left(1, x n^{-1 / 2}\right)+O\left(n^{-3 / 2}\right)
$$

for $x$ and $n$ of the same parity. Each of the $k$ terms in the product part of (6) contributes an extra factor of two in the variance when scaled diffusively, which 
causes the switch from $\beta$ to $\sqrt{2} \beta$ in going from the discrete partition function to the continuum one.

Our proof of Theorem 2.1 essentially follows the strategy that we have already outlined. First we show that, for each fixed $k$, the $k$ th term in the expansion (6) converges to the $k$ th term of the Wiener chaos (7). We find it convenient to use the techniques of $U$-statistics $[21,24,28]$, where such results are the main focus. The general problem begins with an i.i.d. sequence of real-valued random variables $X_{1}, X_{2}, \ldots$, and a symmetric function $f: \mathbb{R}^{k} \rightarrow \mathbb{R}$. One of the main goals is to find limit theorems for sequences of the form

$$
n^{-k \gamma} \sum_{\mathbf{i} \in D_{k}^{n}} f\left(X_{\mathbf{i}_{1}}, \ldots, X_{\mathbf{i}_{k}}\right)
$$

as $n \rightarrow \infty$. Here $f$ is thought of as an observable of $k$ variables, and the summation is over all possible random observations that can be drawn from the set of $n$ variables. A weight function $g: D_{k}^{n} \rightarrow \mathbb{R}$ may be added, leading to the study of sums

$$
n^{-k \gamma} \sum_{\mathbf{i} \in D_{k}^{n}} g(\mathbf{i}) f\left(X_{\mathbf{i}_{1}}, \ldots, X_{\mathbf{i}_{k}}\right) .
$$

This is called an asymmetric statistic, and for each $k$ the terms in (6) have this form. In Section 4 we prove a technical lemma showing that the asymmetric statistics of (6) converge to the multiple stochastic integrals of (7), which is the main step in the proof.

We will also implicitly make use of an extension of $U$-statistics called $U$ processes $[32,33]$. The process is formed by varying the weight functions $g$ through a given set while keeping the realization of the random variables fixed. In this paper we will deal with families of functions $g_{x}$ indexed by $x \in \mathbb{R}$; an asymmetric $U$-process is then given by

$$
x \mapsto n^{-k \gamma} \sum_{\mathbf{i} \in D_{k}^{n}} g_{x}(\mathbf{i}) f\left(X_{\mathbf{i}_{1}}, \ldots, X_{\mathbf{i}_{k}}\right) .
$$

Limiting $U$-process can then be constructed by taking $n \rightarrow \infty$, and the limits take the form of multiple stochastic integrals of white noise over space and time, with the noise weighted by the kernels $g_{x}$.

For our purposes this more general framework is useful for studying the limit of the point-to-point partition function, defined in the following way

$$
Z_{n}^{\omega}\left(x ; \beta n^{-1 / 4}\right)=\mathbf{P}\left[\exp \left\{\beta n^{-1 / 4} H_{n}^{\omega}(S)\right\} \mathbf{1}\left\{S_{n}=x\right\}\right] .
$$

Through this object we can write the polymer endpoint measure as

$$
\mathbf{P}_{n, \beta_{n}}^{\omega}\left(S_{n}=x\right)=\frac{Z_{n}^{\omega}\left(x ; \beta n^{-1 / 4}\right)}{Z_{n}^{\omega}\left(\beta n^{-1 / 4}\right)} .
$$


As above, it is more convenient to consider the modified point-to-point partition function

$$
\begin{aligned}
\mathfrak{Z}_{n}^{\omega}\left(x ; \beta n^{-1 / 4}\right) & :=\mathbf{P}\left[\prod_{i=1}^{n}\left(1+\beta n^{-1 / 4} \omega\left(i, S_{i}\right)\right) \mathbf{1}\left\{S_{n}=x\right\}\right] \\
& =\mathbf{P}\left[\prod_{i=1}^{n}\left(1+\beta n^{-1 / 4} \omega\left(i, S_{i}\right)\right) \mid S_{n}=x\right] p(n, x) .
\end{aligned}
$$

Now the expectation is over walks conditioned to be at $x$ at time $n$, and this changes the Markov transition kernel that weights the noise. As was done in (6), it is easily computed that

$$
\begin{aligned}
& \mathbf{P}\left[\prod_{i=1}^{n}\left(1+\beta n^{-1 / 4} \omega\left(i, S_{i}\right)\right) \mid S_{n}=x\right] \\
& \quad=1+\sum_{k=1}^{n} \beta^{k} n^{-k / 4} \sum_{\mathbf{i} \in D_{k}^{n}} \sum_{\mathbf{x} \in \mathbb{Z}^{k}} p_{x}^{n}(\mathbf{i}, \mathbf{x}) \prod_{j=1}^{k} \omega\left(\mathbf{i}_{j}, \mathbf{x}_{j}\right),
\end{aligned}
$$

where $p_{x}$ is the transition kernel

$$
\begin{aligned}
p_{x}^{n}(\mathbf{i}, \mathbf{x}) & =\mathbf{P}\left(S_{\mathbf{i}_{1}}=\mathbf{x}_{1}, \ldots, S_{\mathbf{i}_{k}}=\mathbf{x}_{k} \mid S_{n}=x\right) \\
& =\frac{p\left(n-\mathbf{i}_{k}, x-\mathbf{x}_{k}\right)}{p(n, x)} \prod_{j=1}^{k} p\left(\mathbf{i}_{j}-\mathbf{i}_{j-1}, \mathbf{x}_{j}-\mathbf{x}_{j-1}\right)
\end{aligned}
$$

for random walks conditioned to be at position $x$ at time $n$. Under diffusive scaling this transition kernel converges to the one for Brownian bridges from zero to a fixed endpoint; the same scaling for the point-to-point partition function leads to:

THEOREM 2.2. The following is true:

- Under the assumption that the $\omega$ have six moments, with mean zero and variance one, the process

$$
x \mapsto \mathbf{P}\left[\prod_{i=1}^{n}\left(1+\beta n^{-1 / 4} \omega\left(i, S_{i}\right)\right) \mid S_{n}=x \sqrt{n}\right]
$$

converges weakly (under the topology of the supremum norm on bounded continuous functions) to the processs

$$
x \mapsto e^{A_{\sqrt{2} \beta}(x)}:=\mathbb{E}\left[: \exp :\left\{\sqrt{2} \beta \int_{0}^{1} W\left(s, X_{s}+x s\right) d s\right\}\right],
$$

where the expectation $\mathbb{E}$ is over Brownian bridges $X_{t}$ that go from zero to zero in time one. See equation (17) for a formal definition of (14). 
- Under the assumption that the $\omega$ satisfy $\lambda(\beta)<\infty$ for $\beta$ sufficiently small, we have

$$
e^{-n \lambda\left(\beta n^{-1 / 4}\right)} \frac{\sqrt{n}}{2} Z_{n}^{\omega}\left(x \sqrt{n} ; \beta n^{-1 / 4}\right) \stackrel{(d)}{\longrightarrow} e^{A \sqrt{2} \beta}(x) \frac{e^{-x^{2} / 2}}{\sqrt{2 \pi}},
$$

where the topology is the supremum norm on bounded continuous functions.

- Under the latter moment assumptions we also have the random local limit theorem

$$
\left\{x \mapsto \frac{\sqrt{n}}{2} \frac{Z_{n}^{\omega}\left(\beta n^{-1 / 4} ; x \sqrt{n}\right)}{Z_{n}^{\omega}\left(\beta n^{-1 / 4}\right)}\right\} \stackrel{(d)}{\longrightarrow}\left\{x \mapsto \frac{1}{\mathcal{Z}_{\sqrt{2} \beta}} e^{A_{\sqrt{2} \beta}(x)} \rho(1, x)\right\},
$$

in the same topology.

REMARK. For the first statement of the theorem the requirement of six moments is purely technical and only used in the proof of the tightness. Convergence of finite dimensional distributions to (14) requires only two moments, and we believe that this is all that should be required for the tightness. This particular six moments assumption, however, is not related to the conjecture for convergence of the $Z_{n}^{\omega}$.

REMARK. The discrete processes in this theorem are technically not continuous functions, but we implicitly assume we are linearly interpolating between the integer values of $x \sqrt{n}$. To keep the notation simple we do not write this.

The $\beta$-indexed family of processes (14) is interesting in its own right, and we understand a significant amount about it. The reasons for writing $A_{\beta}$ in the exponential will soon be apparent. First note that the expectation (14) is to be interpreted, as with (8), as convenient notation for a particular Wiener chaos expansion. In this case the transition kernel for Brownian bridges is used instead of the one for Brownian motion, hence

$$
\begin{aligned}
& e^{A_{\sqrt{2} \beta}(x)} \\
& =1+\sum_{k=0}^{\infty}(\sqrt{2} \beta)^{k} \int_{\Delta_{k}} \int_{\mathbb{R}^{k}} \frac{\varrho\left(1-t_{k}, x-x_{k}\right)}{\varrho(1, x)} \\
& \times \prod_{i=1}^{k} W\left(t_{i}, x_{i}\right) \varrho\left(t_{i}-t_{i-1}, x_{i}-x_{i-1}\right) d t_{i} d x_{i} .
\end{aligned}
$$

This Wiener chaos expansion is the proper definition of how the process varies with $x$, and clearly shows the connection with $U$-processes. As $x$ varies it is the weight function of the white noise that changes and this defines the process. However, the probabilistic shorthand (14), while only formal, provides a much more 
intuitive description than (17). Observe that (14) only uses one Brownian bridge from zero to zero and then simply adds the appropriate drift to create a bridge to a different endpoint. This is, of course, a very simple coupling of Brownian bridges with the same starting point but different endpoints. The simplicity is useful, however, and the shorthand (14) has the advantage of making certain facts immediately obvious, such as:

Proposition 2.3. The process $x \mapsto A_{\beta}(x)$ is stationary.

Proof. Fix $\theta \in \mathbb{R}$, and define $W_{\theta}(t, x):=W(t, x+\theta t)$. As the linear transformation $(t, x) \mapsto(t, x+\theta t)$ has determinant 1 , we immediately have that $W_{\theta}$ is also a white noise on $\mathbb{R}_{+} \times \mathbb{R}$ with the same covariance function as $W$. Then

$$
\begin{aligned}
x \mapsto e^{A_{\beta}(x+\theta)} & =\mathbb{E}\left[: \exp :\left\{\beta \int_{0}^{1} W\left(s, X_{s}+x s+\theta s\right) d s\right\}\right] \\
& =\mathbb{E}\left[: \exp :\left\{\beta \int_{0}^{1} W_{\theta}\left(s, X_{s}+x s\right) d s\right\}\right]
\end{aligned}
$$

has the same law as the original process.

Combining Proposition 2.3 with Theorem 2.2 produces an analogue of a wellknown result from the polynuclear growth model [36]. Taking logarithms of (15) leads to

$$
\begin{aligned}
\log Z_{n}^{\omega}\left(x \sqrt{n} ; \beta n^{-1 / 4}\right)-n \lambda\left(\beta n^{-1 / 4}\right)+\frac{1}{2} \log (n / 4) \\
\rightarrow A_{\sqrt{2} \beta}(x)-\frac{x^{2}}{2}-\log \sqrt{2 \pi} .
\end{aligned}
$$

The right-hand side is a stationary process around a parabola, which is similar to what was first encountered for the PNG droplet [36]. There the stationary process was Airy 2 , and it was shown that it has the Tracy-Widom distribution $F_{\text {GUE }}$ as its one-point marginal distribution. On the level of one-point marginal distributions

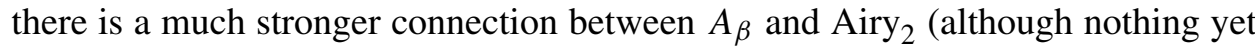
has been rigorously proved on the process level; see [4] for a conjecture). Recent results derived by Amir, Corwin and Quastel [4], and independently by Spohn and Sasamoto [37, 38], give a formula for the one-point marginal distribution of the process $A_{\beta}$ and how this distribution scales as $\beta$ goes to zero or infinity, specifically as $\beta \rightarrow \infty$ the one-point marginals of $A_{\beta}$ converge to those of Airy ${ }_{2}$ :

Proposition 2.4 ([4]). For $\beta>0$ and $x \in \mathbb{R}$,

$$
\begin{aligned}
G_{\beta}(s) & :=\mathbb{P}\left(A_{\sqrt{2} \beta}(x)+2 \beta^{4} / 3 \leq s\right) \\
& =1-\int e^{-e^{-r}} f\left(s-\log \left(32 \pi \beta^{4}\right) / 2-r\right) d r,
\end{aligned}
$$


with

$$
f(r)=\kappa_{\beta}^{-1} \operatorname{det}\left(I-K_{\sigma_{\beta}}\right) \operatorname{tr}\left(\left(I-K_{\sigma_{\beta}}\right)^{-1} P_{\text {Airy }}\right),
$$

where $\kappa_{\beta}=2 \beta^{4 / 3}$, and $K_{\sigma_{\beta}}$ and $P_{\text {Airy }}$ are operators acting on $L^{2}\left(\kappa_{\beta}^{-1} r, \infty\right)$ given by their kernels

$$
\begin{aligned}
P_{\text {Airy }}(x, y) & =\operatorname{Ai}(x) \operatorname{Ai}(y), \\
K_{\sigma_{\beta}}(x, y) & =\text { P.V. } \int \sigma_{\beta}(t) \operatorname{Ai}(x+t) \operatorname{Ai}(y+t) d t,
\end{aligned}
$$

with $\sigma_{\beta}(t)=\left(1-e^{-\kappa_{\beta} t}\right)^{-1}$. Moreover, the distribution functions $G_{\beta}$ satisfy the asymptotic relations

$$
G_{\beta}\left(2^{4 / 3} \beta^{4 / 3} s\right) \stackrel{\beta \rightarrow \infty}{\longrightarrow} F_{\mathrm{GUE}}\left(2^{1 / 3} s\right)
$$

and

$$
G_{\beta}\left(2^{1 / 2} \pi^{1 / 4} \beta s\right) \stackrel{\beta \rightarrow 0}{\longrightarrow} \int_{-\infty}^{s} \frac{e^{-x^{2} / 2}}{\sqrt{2 \pi}} d x .
$$

This result is derived using steepest descent analysis on a Tracy-Widom formula [41-43] for the Asymmetric Simple Exclusion Process (ASEP). The exact form of the $G_{\beta}$ distributions are not used in this paper, but the asymptotics are important and suggest how the polymer scalings behave as $\beta \rightarrow \infty$.

In particular, combining (18), (19) and (20), we have the following corollary, which is the first general result for Tracy-Widom asymptotics for polymers at nonzero temperature:

COROLLARY 2.5 (Weak universality for directed random polymers in $1+1$ dimensions). Assume that $\omega$ are i.i.d. with $\lambda(\beta)<\infty$ for $\beta$ sufficiently small. Then as $n \rightarrow \infty$ followed by $\beta \rightarrow \infty$,

$$
\frac{\log Z_{n}^{\omega}\left(0 ; \beta n^{-1 / 4}\right)-n \lambda\left(\beta n^{-1 / 4}\right)+\log \sqrt{\pi n / 2}+2 \beta^{4} / 3}{2 \beta^{4 / 3}} \stackrel{(d)}{\longrightarrow} F_{\text {GUE }} .
$$

In the concluding remarks of Section 7 we discuss our conjecture that this statement holds whenever the $\omega$ have six moments, and is false otherwise. The corollary above should be compared to the following conjecture (see [9] for the 5 moment assumption):

CONJECTURe 2.6 (Strong universality for directed random polymers in $1+1$ dimensions). Assume that $\omega$ are i.i.d. with 5 moments. Then there are constants $c=c(\beta)$ and $\sigma=\sigma(\beta)$ such that as $n \rightarrow \infty$,

$$
\frac{\log Z_{n}^{\omega}(0 ; \beta)-c(\beta) n}{\sigma(\beta) n^{1 / 3}} \stackrel{(d)}{\longrightarrow} F_{\mathrm{GUE}}
$$

for all $\beta>0$. 
Finally we consider the transition probabilities for the polymer. It is worth emphasizing that, given the environment field $\omega$, the polymer measure $\mathbf{P}_{n, \beta}^{\omega}$ is Markov, and therefore it is uniquely determined by its transition probabilities. These transition probabilities are functions of the environment, and the inhomogeneous nature of the environment means that the probabilities are inhomogeneous in space and time. For $0 \leq m<k \leq n$ and $x, y \in \mathbb{Z}$ we define the four-parameter field $Z^{\omega}(m, y ; k, x ; \beta)$ by

$$
Z^{\omega}(m, y ; k, x ; \beta)=\mathbf{P}\left[\exp \left\{\beta \sum_{i=m+1}^{k} \omega\left(i, S_{i}\right)\right\} \mathbf{1}\left\{S_{k}=x\right\} \mid S_{m}=y\right],
$$

which is a point-to-point partition function for a polymer starting at position $y$ at time $m$. For the point-to-line versions we introduce the notation

$$
Z^{\omega}(m, y ; k, * ; \beta)=\sum_{x \in \mathbb{Z}} Z^{\omega}(m, y ; k, x ; \beta) .
$$

It is then straightforward to verify that for $0 \leq m<k \leq n$ the polymer has transition probabilities given by

$$
\begin{aligned}
\mathbf{P}_{n, \beta}^{\omega} & \left(S_{i+1}=S_{i} \pm 1 \mid S_{1}, \ldots, S_{i}\right) \\
& =\frac{1}{2} e^{\beta \omega\left(i+1, S_{i} \pm 1\right)} \frac{Z^{\omega}\left(i+1, S_{i} \pm 1 ; n, * ; \beta\right)}{Z^{\omega}\left(i, S_{i} ; n, * ; \beta\right)} .
\end{aligned}
$$

The polymer is clearly Markov since the equation on the right only depends on $S_{i}$. More importantly though, this equation shows that the four-parameter field uniquely determines the polymer measure. Our final theorem describes its scaling limit as $n \rightarrow \infty$. As before we initially work with the modified partition function

$$
\mathfrak{Z}^{\omega}(m, y ; k, x ; \beta)=\mathbf{P}\left[\prod_{i=m+1}^{k}\left(1+\beta \omega\left(i, S_{i}\right)\right) \mathbf{1}\left\{S_{k}=x\right\} \mid S_{m}=y\right]
$$

and then transfer the results to the usual exponential form.

THEOREM 2.7. The following is true:

- Assuming that the $\omega$ have six moments with mean zero and variance one, the fields

$$
(s, y ; t, x) \rightarrow \frac{\sqrt{n}}{2} \mathfrak{Z}^{\omega}\left(n s, y \sqrt{n} ; n t, x \sqrt{n} ; \beta n^{-1 / 4}\right)
$$

converge weakly as $n \rightarrow \infty$ to a random field $\mathcal{Z}_{\sqrt{2} \beta}(s, y ; t, x)$. The topology is the sup norm on bounded continuous functions with the domain $\{(s, y ; t, x): 0 \leq$ $s<t \leq 1, x, y \in \mathbb{R}\}$. 
- Assuming that $\lambda(\beta)<\infty$ for small $\beta$, the fields

$$
(s, y ; t, x) \mapsto \frac{\sqrt{n}}{2} e^{-n(t-s) \lambda\left(\beta n^{-1 / 4}\right)} Z^{\omega}\left(n s, y \sqrt{n} ; n t, x \sqrt{n} ; \beta n^{-1 / 4}\right)
$$

converge weakly to the same limit, under the same topology.

- The limiting field $\mathcal{Z}_{\beta}(s, y ; t, x)$ has the chaos representation

$$
\begin{array}{rl}
\mathcal{Z}_{\beta}(s, y ; t, x)=\varrho(s, y ; t, x) & \\
+\sum_{k=1}^{\infty} \beta^{k} \int_{\Delta_{k}(s, t]} \int_{\mathbb{R}^{k}} \prod_{i=1}^{k} & W\left(t_{i}, x_{i}\right) \\
& \times \varrho\left(t_{i}-t_{i-1}, x_{i}-x_{i-1}\right) \\
& \times \varrho\left(t-t_{k}, x-x_{k}\right) d x_{i} d t_{i},
\end{array}
$$

with $\Delta_{k}(s, t]=\left\{s=t_{0}<t_{1}<\cdots<t_{k}<t\right\}$, and $x_{i} \in \mathbb{R}$ with $x_{0}=y$.

REMARK. As in the remarks after Theorem 2.2, the six moments assumption for $\mathfrak{Z}_{n}^{\omega}$ is only used in the tightness, but we do not believe it to be necessary. Convergence of the finite dimensional distributions of $\mathfrak{Z}_{n}^{\omega}$ goes through with only two moments. Also, the discrete fields are defined only on the mesh where ( $n s, y \sqrt{n} ; n t, x \sqrt{n}$ ) take integer values, but we use a linear interpolation scheme to extend it to the whole space. A particular method is outlined in the Appendix.

REMARK. As is discussed in the companion paper [2], the four-parameter field is the chaos solution to the stochastic partial differential equation

$$
\partial_{t} \mathcal{Z}_{\beta}=\frac{1}{2} \partial_{x x} \mathcal{Z}_{\beta}+\beta W \mathcal{Z}_{\beta}, \quad \mathcal{Z}_{\beta}(s, y ; s, x)=\delta_{0}(x-y) .
$$

This is the stochastic heat equation with multiplicative noise. The logarithm of this field is the Hopf-Cole solution of the so-called KPZ equation [4, 6, 29]. The continuum analogue of the field (22) is the point-to-line partition function defined by

$$
\mathcal{Z}_{\beta}(s, y ; t, *)=\int \mathcal{Z}_{\beta}(s, y ; t, x) d x
$$

REMARK. From equation (23) it can easily be derived that the multi-step polymer transition probabilities are

$$
\mathbf{P}_{n, \beta}^{\omega}\left(S_{k}=x \mid S_{m}=y\right)=\frac{Z^{\omega}(m, y ; k, x ; \beta) Z^{\omega}(k, x ; n, * ; \beta)}{Z^{\omega}(m, y ; n, * ; \beta)} .
$$

Combining this with Theorems 2.1 and 2.2 leads to the statement in the Introduction that

$$
\frac{\sqrt{n}}{2} \mathbf{P}_{n, \beta_{n}}^{\omega}\left(S_{n t}=x \sqrt{n} \mid S_{n s}=y \sqrt{n}\right) \stackrel{(d)}{\longrightarrow} \frac{\mathcal{Z}_{\sqrt{2} \beta}(s, y ; t, x) \mathcal{Z}_{\sqrt{2} \beta}(t, x ; 1, *)}{\mathcal{Z}_{\sqrt{2} \beta}} .
$$




\section{Wiener chaos.}

3.1. Brownian motion and simple random walk. Throughout we let $S_{n}$ be a simple random walk on $\mathbb{Z}$ and $B_{t}$ be a standard Brownian motion on $\mathbb{R}$. For $i \in \mathbb{N}$ or $t \geq 0$ and $x \in \mathbb{R}$ let

$$
p(i, x)=\mathbf{P}\left(S_{i}=x\right), \quad \varrho(t, x)=\frac{e^{-x^{2} / 2 t}}{\sqrt{2 \pi t}} .
$$

We will make heavy use of the joint probability densities of both simple random walk and Brownian motion, for which we introduce the notation

$$
p_{k}(\mathbf{i}, \mathbf{x})=\mathbf{P}\left(S_{\mathbf{i}_{1}}=\mathbf{x}_{1}, \ldots, S_{\mathbf{i}_{k}}=\mathbf{x}_{k}\right)=\prod_{j=1}^{k} p\left(\mathbf{i}_{j}-\mathbf{i}_{j-1}, \mathbf{x}_{j}-\mathbf{x}_{j-1}\right)
$$

for $\mathbf{i} \in D_{k}^{n}, \mathbf{x} \in \mathbb{R}^{k}$. Here $D_{k}^{n}$ is the integer simplex

$$
D_{k}^{n}=\left\{\mathbf{i} \in[n]^{k}: 1 \leq \mathbf{i}_{1}<\mathbf{i}_{2}<\cdots<\mathbf{i}_{k} \leq n\right\},
$$

with $[n]:=\{1,2, \ldots, n\}$. For $\mathbf{i} \in \mathbb{Z}^{k}$ we sometimes write $|\mathbf{i}|$ as shorthand for the length $k$ of the vector.

The parity property of simple random walk, that it is only on the even integers at even times and the odd integers at odd times, plays a role in much of the technical analysis. We write $i \leftrightarrow x$ if $i$ and $x$ are of the same parity, and for $\mathbf{i} \in D_{k}^{n}, \mathbf{x} \in \mathbb{Z}^{k}$ we write $\mathbf{i} \leftrightarrow \mathbf{x}$ if $\mathbf{i}_{j} \leftrightarrow \mathbf{x}_{j}$ for $1 \leq j \leq k$. Given $x \in \mathbb{R}$ and $i \in \mathbb{N}$ we define $[x]_{i}$ to be the closest element of $\mathbb{Z}$ to $x$ such that $i \leftrightarrow x$. For a point $\mathbf{x} \in \mathbb{R}^{k}$ and $\mathbf{i} \in D_{k}^{n}$ define $[\mathbf{x}]_{\mathbf{i}} \in \mathbb{Z}^{k}$ by $\left([\mathbf{x}]_{\mathbf{i}}\right)_{k}=\left[x_{k}\right]_{i_{k}}$. It will often be useful to extend $p$ to all of $\mathbb{R}^{k}$, so we also define

$$
\bar{p}_{k}(\mathbf{i}, \mathbf{x})=2^{-k} p_{k}\left(\mathbf{i},[\mathbf{x}]_{\mathbf{i}}\right) .
$$

The $2^{-|\mathbf{i}|}$ factor normalizes $\bar{p}(\mathbf{i}, \cdot)$ to be a probability measure on $\mathbb{R}^{k}$. It is useful to observe that the $\bar{p}(\mathbf{i}, \mathbf{x})$ are the finite dimensional distributions for the random walk $X_{n}=S_{n}+U_{n}$, where $\left\{U_{i}\right\}_{i \geq 1}$ is a sequence of independent random variables uniformly distributed on $(-1,1)$.

For Brownian motion we use the analogous notation

$$
\varrho_{k}(\mathbf{t}, \mathbf{x})=\prod_{j=1}^{k} \varrho\left(\mathbf{t}_{j}-\mathbf{t}_{j-1}, \mathbf{x}_{j}-\mathbf{x}_{j-1}\right)
$$

for $\mathbf{t}$ in the simplex

$$
\Delta_{k}=\left\{\mathbf{t} \in \mathbb{R}^{k}: 0 \leq \mathbf{t}_{1}<\mathbf{t}_{2}<\cdots<\mathbf{t}_{k} \leq 1\right\} .
$$


3.2. White noise and stochastic integration on $[0,1] \times \mathbb{R}$. In this section we briefly recall the elementary theory of white noise and stochastic integration on the particular measure space $L^{2}([0,1] \times \mathbb{R}, \mathcal{B}, d t d x)$. Here $\mathcal{B}$ is the $\sigma$-algebra of Borel subsets, and $d t d x$ denotes Lebesgue measure on the space. We let $\mathcal{B}_{f}$ be the subset of $\mathcal{B}$ consisting of sets of finite Lebesgue measure. Observe that $\mathcal{B}=\sigma\left(\mathcal{B}_{f}\right)$ because Lebesgue measure is $\sigma$-finite on the given space.

A white noise on $[0,1] \times \mathbb{R}$ is a collection of mean zero Gaussian random variables defined on a common probability space $\left(\Omega_{W}, \mathcal{F}_{W}, \mathbb{Q}\right)$ and indexed by $\mathcal{B}_{f}$

$$
W=\left\{W(A): A \in \mathcal{B}_{f}\right\} .
$$

This means that every finite collection of the form $\left(W\left(A_{1}\right), \ldots, W\left(A_{k}\right)\right)$ has a $k$ dimensional Gaussian distribution, with mean zero and covariance structure given by

$$
\mathrm{E}[W(A) W(B)]=|A \cap B| .
$$

In particular if $A$ and $B$ are disjoint then $W(A)$ and $W(B)$ are independent.

For $g \in L^{2}([0,1] \times \mathbb{R}, \mathcal{B}, d t d x)$ the stochastic integral

$$
I_{1}(g)=\int_{0}^{1} \int g(t, x) W(d t d x)
$$

is constructed by first defining $I_{1}$ on simple functions via

$$
I_{1}\left(\sum_{i=1}^{n} \alpha_{i} \mathbf{1}_{A_{i}}\right)=\sum_{i=1}^{n} \alpha_{i} W\left(A_{i}\right)
$$

where $A_{i} \in \mathcal{B}_{f}$, and then using the density of simple functions in $L^{2}([0,1] \times \mathbb{R})$ and the completeness of $L^{2}\left(\Omega_{W}, \mathcal{F}_{W}, \mathbb{Q}\right)$ to define $I_{1}(g)$. The construction shows that $I_{1}$ is linear in the sense that for all $\alpha_{1}, \ldots, \alpha_{n} \in \mathbb{R}$ and $g_{1}, \ldots, g_{n} \in L^{2}([0,1] \times$ $\mathbb{R})$ we have, with probability one,

$$
I_{1}\left(\sum_{i=1}^{n} \alpha_{i} g_{i}\right)=\sum_{i=1}^{n} \alpha_{i} I_{1}\left(g_{i}\right) .
$$

For each $g$ we have that $I_{1}(g) \sim N\left(0,\|g\|_{L^{2}}^{2}\right)$, and moreover $I_{1}$ preserves the Hilbert space structure of $L^{2}([0,1] \times \mathbb{R})$,

$$
\mathrm{E}\left[I_{1}(g) I_{1}(h)\right]=\int_{0}^{1} \int g(t, x) h(t, x) d t d x .
$$

Now we define multiple stochastic integrals on $L^{2}\left([0,1]^{k} \times \mathbb{R}^{k}\right)$ for $k>1$. For notation we use

$$
I_{k}(g)=\int_{[0,1]^{k}} \int_{\mathbb{R}^{k}} g(\mathbf{t}, \mathbf{x}) W^{\otimes k}(d \mathbf{t} d \mathbf{x})
$$


for $g \in L^{2}\left([0,1]^{k} \times \mathbb{R}^{k}\right)$. The construction is similar to the $k=1$ case except that mild care must be given to integration along the "diagonals" of the space. Moreover, the stochastic integral is only truly defined for symmetric functions on $[0,1]^{k} \times \mathbb{R}^{k}$. Here $g$ is symmetric if $g(\mathbf{t}, \mathbf{x})=g(\pi \mathbf{t}, \pi \mathbf{x})$ for all $(\mathbf{t}, \mathbf{x}) \in[0,1]^{k} \times \mathbb{R}^{k}$ and $\pi \in S_{k}$, the group of permutations on $\{1,2, \ldots, k\}$. The permutations act on vectors in the obvious way: $\pi \mathbf{t}=\left(\mathbf{t}_{\pi 1}, \ldots, \mathbf{t}_{\pi k}\right)$ and $\pi \mathbf{x}$ defined similarly. We let $L_{S}^{2}\left([0,1]^{k} \times \mathbb{R}^{k}\right)$ denote the subspace of symmetric functions in $L^{2}$. As in the $k=1$ case it is enough to define $I_{k}$ on a dense subset of $L_{S}^{2}$ from which it can be linearly extended (in a unique way) to the entire space. One dense subset is the functions of the form

$$
g(\mathbf{t}, \mathbf{x})=\sum_{\pi \in S_{k}} \prod_{j=1}^{k} \mathbf{1}\left\{\left(\mathbf{t}_{\pi j}, \mathbf{x}_{\pi j}\right) \in A_{j}\right\},
$$

where the $A_{j}, j=1, \ldots, k$ are disjoint subsets of $[0,1] \times \mathbb{R}$. We define

$$
I_{k}(g)=k ! \prod_{j=1}^{k} W\left(A_{j}\right)
$$

It is standard to show that there exists a unique linear extension of $I_{k}$ from functions of the form (25) onto $L_{S}^{2}$ such that each $I_{k}(g)$ is a mean zero random variable with variance $\|g\|_{L^{2}}^{2}$, and the covariance structure is

$$
\mathrm{E}\left[I_{k}(g) I_{k}(h)\right]=\langle g, h\rangle_{L^{2}\left([0,1]^{k} \times \mathbb{R}^{k}\right)} .
$$

Moreover, relation (26) can be extended to show that if $g_{1}, \ldots, g_{k} \in L^{2}([0,1] \times \mathbb{R})$ are all orthogonal to each other, then

$$
I_{k}\left(\sum_{\pi \in S_{k}} \prod_{j=1}^{k} g_{j}\left(\mathbf{t}_{\pi j}, \mathbf{x}_{\pi j}\right)\right)=k ! \prod_{j=1}^{k} I_{1}\left(g_{j}\right) .
$$

Finally, we also adopt the convention that $I_{k}$ extends to nonsymmetric functions $g \in L^{2}\left([0,1]^{k} \times \mathbb{R}^{k}\right)$ via symmetrization. We define

$$
I_{k}(g)=I_{k}(\operatorname{Sym} g) \text {, }
$$

where

$$
\operatorname{Sym} g(\mathbf{t}, \mathbf{x})=\frac{1}{k !} \sum_{\pi \in S_{k}} g(\pi \mathbf{t}, \pi \mathbf{x}) .
$$

REMARK. Suppose that $g: \Delta_{k} \times \mathbb{R}^{k} \rightarrow \mathbb{R}$ instead. We extend it to a function on $[0,1]^{k} \times \mathbb{R}^{k}$ by defining it to be zero for $\mathbf{t} \notin \Delta_{k}$. Then

$$
I_{k}(g)=\int_{[0,1]^{k}} \int_{\mathbb{R}^{k}} \operatorname{Sym} g(\mathbf{t}, \mathbf{x}) W^{\otimes k}(d \mathbf{t} d \mathbf{x})=k ! \int_{\Delta_{k}} \int_{\mathbb{R}^{k}} g(\mathbf{t}, \mathbf{x}) W^{\otimes k}(d \mathbf{t} d \mathbf{x})
$$

since symmetrizing simply "copies" the functions into the $k$ ! permutations of $\Delta_{k}$ that make up $[0,1]^{k}$, ignoring the diagonals which do not affect the stochastic integral anyways. 
3.3. Wiener chaos on $[0,1] \times \mathbb{R}$. In the context of this paper Wiener chaos may be regarded as a way of representing random variables as infinite sums of multiple stochastic integrals. For every random variable $X \in L^{2}\left(\Omega_{W}, \mathcal{F}_{W}, \mathbb{Q}\right)$, the Wiener chaos decomposition states that there is a unique sequence of symmetric functions $g_{k} \in L_{S}^{2}\left([0,1]^{k} \times \mathbb{R}^{k}\right), k \geq 1$, such that

$$
X=\sum_{k=0}^{\infty} I_{k}\left(g_{k}\right) .
$$

Here $g_{0}$ is simply a constant and $I_{0}\left(g_{0}\right)=g_{0}$. In fact as the $k \geq 1$ terms of the chaos series are all mean zero, $g_{0}$ must be the mean of $X$. Moreover, by the orthogonality of $I_{k_{1}}\left(g_{1}\right)$ and $I_{k_{2}}\left(g_{2}\right)$ for $k_{1} \neq k_{2}$ we have the relation

$$
\mathrm{E}\left[X^{2}\right]=\sum_{k=0}^{\infty}\left\|g_{k}\right\|_{L^{2}\left([0,1]^{k} \times \mathbb{R}^{k}\right)}^{2} .
$$

The situation works in reverse also. Given any element of the symmetric Fock space over $L^{2}([0,1] \times \mathbb{R})$, that is,

$$
g=\left(g_{0}, g_{1}, g_{2}, \ldots\right) \in \bigoplus_{k=0}^{\infty} L_{S}^{2}\left([0,1]^{k} \times \mathbb{R}^{k}\right),
$$

the map $I: \bigoplus_{k=0}^{\infty} L_{S}^{2}\left([0,1]^{k} \times \mathbb{R}^{k}\right) \rightarrow L^{2}\left(\Omega_{W}, \mathcal{F}_{W}, \mathbb{Q}\right)$ defined by $I(g)=$ $\sum_{k \geq 0} I_{k}\left(g_{k}\right)$ is an isometry. The norm on the Fock space is

$$
\|g\|_{F}^{2}=\sum_{k=0}^{\infty}\left\|g_{k}\right\|_{L^{2}\left([0,1]^{k} \times \mathbb{R}^{k}\right)}^{2} .
$$

3.4. Wiener chaos for Brownian transition probabilities. The Brownian transition probabilities are easily shown to define an element of the Fock space $\bigoplus_{k \geq 0} L^{2}\left(\Delta_{k} \times \mathbb{R}^{k}\right)$. Define

$$
\varrho(\beta)=\left(1, \beta \varrho_{1}, \beta^{2} \varrho_{2}, \ldots\right),
$$

where the $\varrho_{k}$ are defined in Section 2.1. For all $\beta \in \mathbb{R}$ it is easily computed that $\varrho(\beta)$ belongs to the Fock space, since

$$
\varrho_{k}(\mathbf{t}, \mathbf{x})^{2}=\varrho_{k}(\mathbf{t}, \sqrt{2} \mathbf{x}) \prod_{j=1}^{k} \frac{1}{\sqrt{2 \pi\left(\mathbf{t}_{j}-\mathbf{t}_{j-1}\right)}},
$$

and hence

$$
\begin{aligned}
\int_{\Delta_{k}} \int_{\mathbb{R}^{k}} \varrho_{k}(\mathbf{t}, \mathbf{x})^{2} d \mathbf{x} d \mathbf{t} & =(4 \pi)^{-k / 2} \int_{\Delta_{k}} \prod_{j=1}^{k} \frac{1}{\sqrt{\mathbf{t}_{j}-\mathbf{t}_{j-1}}} d \mathbf{t} \\
& =(4 \pi)^{-k / 2} B\left(\frac{1}{2}, \frac{1}{2}, \ldots, \frac{1}{2}, 1\right)=\frac{1}{2^{k} \Gamma((k / 2)+1)} .
\end{aligned}
$$


The second equality comes from recognizing that the integrand is the density of the Dirichlet distribution, for which the beta function $B$ is the normalizing constant. Recalling that the beta function is the ratio of Gamma functions [1] produces the final expression, and the extremely fast decay of this expression in $k$ clearly shows that $\|\varrho(\beta)\|_{F}^{2}<\infty$ for all $\beta \in \mathbb{R}$. This gives the following:

LEMMA 3.1. The Wiener chaos $\mathcal{Z}_{\beta}$ has the representation $\mathcal{Z}_{\beta}=I(\varrho(\beta))$.

For fixed $y \in \mathbb{R}$ we also define

$$
\varrho_{k}(\mathbf{t}, \mathbf{x} ; y)=\varrho_{k}(\mathbf{t}, \mathbf{x}) \varrho\left(1-\mathbf{t}_{k}, y-\mathbf{x}_{k}\right)
$$

for which it can be computed in a similar manner as above that

$$
\begin{array}{r}
\int_{\Delta_{k}} \int_{\mathbb{R}^{k}} \varrho_{k}(\mathbf{t}, \mathbf{x} ; y)^{2} d \mathbf{x} d \mathbf{t} \\
=\frac{e^{-y^{2}}}{2^{k+1 / 2} \Gamma((k+1) / 2)} .
\end{array}
$$

We define $\varrho(\beta ; y)=\left(1, \beta \varrho_{1}(\cdot, \cdot ; y), \beta^{2} \varrho_{2}(\cdot, \cdot ; y), \ldots\right)$, and then clearly $\sup _{y} \| \varrho(\beta$; $y) \|_{F}^{2}<\infty$ uniformly in $y$, for all $\beta \in \mathbb{R}$.

4. $\boldsymbol{U}$-Statistics. In this section we prove the main technical theorem for convergence in law of the partition functions. The results presented here are standard in the theory of $U$-statistics. We are mostly interested in sums of the form

$$
\sum_{\mathbf{i} \in D_{k}^{n}} \sum_{\substack{\mathbf{x} \in \mathbb{Z}^{k} \\ \mathbf{i} \leftrightarrow \mathbf{x}}} g(\mathbf{i}, \mathbf{x}) \omega(\mathbf{i}, \mathbf{x})
$$

for some weight function $g$; this particular type of sum is generally referred to as a weighted or asymmetric $U$-statistic.

4.1. U-Statistics for space-time random environments. It will actually be more efficient to slightly generalize our results to sums over unordered $\mathbf{i}$, that is, $\mathbf{i} \in E_{k}^{n}$ where

$$
E_{k}^{n}=\left\{\mathbf{i} \in[n]^{k}: \mathbf{i}_{j} \neq \mathbf{i}_{l} \text { for } j \neq l\right\} .
$$

Furthermore the theory will be easier to work with when the weight function is extracted from an $L^{2}$ function on $[0,1]^{k} \times \mathbb{R}^{k}$. We first discretize such a function by replacing it by its average on rectangles; we use rectangles of the form

$$
\mathcal{R}_{k}^{n}:=\left\{\left(\frac{\mathbf{i}-\mathbf{1}}{n}, \frac{\mathbf{i}}{n}\right] \times\left(\frac{\mathbf{x}-\mathbf{1}}{\sqrt{n}}, \frac{\mathbf{x}+\mathbf{1}}{\sqrt{n}}\right]: \mathbf{i} \in D_{k}^{n}, \mathbf{x} \in \mathbb{Z}^{k}, \mathbf{i} \leftrightarrow \mathbf{x}\right\},
$$


with 1 being the vector of all ones. Observe that $|R|=2^{k} n^{-3 k / 2}$ for $R \in \mathcal{R}_{k}^{n}$. For $g \in L^{2}\left([0,1]^{k} \times \mathbb{R}^{k}\right)$ define $\bar{g}_{n}$ by

$$
\bar{g}_{n}(\mathbf{t}, \mathbf{x})=\frac{1}{|R|} \int_{R} g \quad(\mathbf{t}, \mathbf{x}) \in R \in \mathcal{R}_{n} .
$$

In probabilistic terms $\bar{g}_{n}$ is simply the conditional expectation of $g$ onto the rectangles of $\mathcal{R}_{k}^{n}$. Now we define weighted $U$-statistics via

$$
\mathcal{S}_{k}^{n}(g)=2^{k / 2} \sum_{\mathbf{i} \in E_{k}^{n}} \sum_{\mathbf{x} \in \mathbb{Z}^{k}} \bar{g}_{n}\left(\frac{\mathbf{i}}{n}, \frac{\mathbf{x}}{\sqrt{n}}\right) \mathbf{1}\{\mathbf{i} \leftrightarrow \mathbf{x}\} \omega(\mathbf{i}, \mathbf{x}) .
$$

REMARK. Observe that in the space direction the rectangles have length $2 / \sqrt{n}$ rather than $1 / \sqrt{n}$. This is one way of dealing with the periodicity issue of simple random walk.

From this definition we have the following:

LEMMA 4.1. The map $\mathcal{S}_{k}^{n}$ is linear in the sense that for all $\alpha_{1}, \ldots, \alpha_{m} \in \mathbb{R}$ and $g_{1}, \ldots, g_{m} \in L^{2}\left([0,1]^{k} \times \mathbb{R}^{k}\right)$ we have

$$
\sum_{l=1}^{m} \alpha_{l} \mathcal{S}_{k}^{n}\left(g_{l}\right)=\mathcal{S}_{k}^{n}\left(\sum_{l=1}^{m} \alpha_{l} g_{l}\right),
$$

with probability one. For all $k$ the variables $\mathcal{S}_{k}^{n}(g)$ are mean zero, and for $k_{1} \neq k_{2}$ and $g_{i} \in L^{2}\left([0,1]^{k_{i}} \times \mathbb{R}^{k_{i}}\right)$ we have

$$
Q\left[\mathcal{S}_{k_{1}}^{n}\left(g_{1}\right) \mathcal{S}_{k_{2}}^{n}\left(g_{2}\right)\right]=0 .
$$

For $k_{1}=k_{2}=k$ we have

$$
Q\left[\mathcal{S}_{k}^{n}(g)^{2}\right] \leq n^{3 k / 2}\left\|\bar{g}_{n}\right\|_{L^{2}\left([0,1]^{k} \times \mathbb{R}^{k}\right)}^{2} \leq n^{3 k / 2}\|g\|_{L^{2}\left([0,1]^{k} \times \mathbb{R}^{k}\right)}^{2} .
$$

PROOF. The linearity and mean zero properties are obvious. For the covariance relation for $k_{1} \neq k_{2}$, simply observe that if $\mathbf{i} \in E_{k_{1}}^{n}, \mathbf{x} \in \mathbb{Z}^{k_{1}}, \mathbf{i}^{\prime} \in E_{k_{2}}^{n}, \mathbf{x}^{\prime} \in \mathbb{Z}^{k_{1}}$, then

$$
Q\left[\prod_{j_{1}=1}^{k_{1}} \omega\left(\mathbf{i}_{j_{1}}, \mathbf{x}_{j_{1}}\right) \prod_{j_{2}=1}^{k_{2}} \omega\left(\mathbf{i}_{j_{2}}^{\prime}, \mathbf{x}_{j_{2}}^{\prime}\right)\right]=0
$$

since there is necessarily one $\omega$ term that is distinct from all others, and its independence from the rest implies zero expectation. In the $k_{1}=k_{2}=k$ case observe that

$$
Q\left[\prod_{j=1}^{k} \omega\left(\mathbf{i}_{j}, \mathbf{x}_{j}\right) \prod_{j=1}^{k} \omega\left(\mathbf{i}_{j}^{\prime}, \mathbf{x}_{j}^{\prime}\right)\right]=\mathbf{1}\left\{\mathbf{i}=\mathbf{i}^{\prime}, \mathbf{x}=\mathbf{x}^{\prime}\right\}
$$


Hence

$$
\begin{aligned}
Q\left[\mathcal{S}_{k}^{n}(g)^{2}\right] & =2^{k} \sum_{\mathbf{i} \in E_{k}^{n}} \sum_{\mathbf{x} \in \mathbb{Z}^{k}} \bar{g}_{n}\left(\frac{\mathbf{i}}{n}, \frac{\mathbf{x}}{\sqrt{n}}\right)^{2} \\
& \leq 2^{k} \sum_{\mathbf{i} \in[n]^{k}} \sum_{\mathbf{x} \in \mathbb{Z}^{k}} \frac{\mathbf{1}\{\mathbf{i} \leftrightarrow \mathbf{x}\}}{|R|} \int_{R} g(\mathbf{t}, \mathbf{y})^{2} d \mathbf{t} d \mathbf{y} \\
& =n^{3 k / 2} \int_{[0,1]^{k}} \int_{\mathbb{R}^{k}} g(\mathbf{t}, \mathbf{y})^{2} d \mathbf{t} d \mathbf{y} .
\end{aligned}
$$

The inequality is an application of the Cauchy-Schwarz lemma, or simply from the fact that $\bar{g}_{n}$ is a conditional expectation of $g$.

We next state a standard weak convergence result that we will make repeated use of. A proof can be found in [7], Chapter 1, Theorem 4.2.

LEMMA 4.2. Let $Y_{k}^{n}, Y_{k}, Y^{n}, Y$ be real-valued random variables, and assume that for each fixed $n$ the $Y_{k}^{n}$ and $Y^{n}$ are defined on a common probability space. Assume that the following diagram holds:

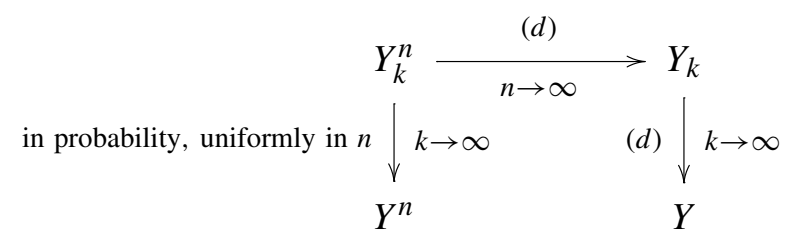

Then $Y^{n} \stackrel{(d)}{\longrightarrow} Y$.

The following is one of our main technical theorems. The proof borrows heavily from [28], Theorem 11.16.

THEOREM 4.3. Let $g \in L^{2}\left([0,1]^{k} \times \mathbb{R}^{k}\right)$. Then, as $n \rightarrow \infty$,

$$
n^{-3 k / 4} \mathcal{S}_{k}^{n}(g) \stackrel{(d)}{\longrightarrow} \int_{[0,1]^{k}} \int_{\mathbb{R}^{k}} g(\mathbf{t}, \mathbf{x}) W^{\otimes k}(d \mathbf{t} d \mathbf{x}) .
$$

Moreover for any finite collection of $k_{1}, \ldots, k_{m} \in \mathbb{N}$ and $g_{1}, \ldots, g_{m}$ with $g_{i} \in$ $L^{2}\left([0,1]^{k_{i}} \times \mathbb{R}^{k_{i}}\right)$, one has the joint convergence

$$
\left(n^{-3 k_{1} / 4} \mathcal{S}_{k_{1}}^{n}\left(g_{1}\right), \ldots, n^{-3 k_{m} / 4} \mathcal{S}_{k_{m}}^{n}\left(g_{m}\right)\right) \stackrel{(d)}{\longrightarrow}\left(I_{k_{1}}\left(g_{1}\right), \ldots, I_{k_{m}}\left(g_{m}\right)\right)
$$

PROOF. The proof proceeds in several steps, beginning with simple functions $g$ for the $k=1$ case and then bootstrapping to the general case via a density argument. 
Step 1. Let $k=1$ and assume that $g(t, x)=\mathbf{1}\left\{t_{0}<t \leq t_{1}, x_{0}<x \leq x_{1}\right\}$. Then

$$
\mathcal{S}_{1}^{n}(g)=2^{1 / 2} \sum_{n t_{0}<i \leq n t_{1}} \sum_{\sqrt{n} x_{0}<x \leq \sqrt{n} x_{1}} \mathbf{1}\{i \leftrightarrow x\} \omega(i, x)
$$

is the sum of order $n^{3 / 2}\left(t_{1}-t_{0}\right)\left(x_{1}-x_{0}\right)+O(n)$ mean zero, variance one random variables [the $2^{1 / 2}$ in front counters the cancelation of half the $\omega(i, x)$ terms through the $i \leftrightarrow x$ condition], and hence by the central limit theorem

$$
n^{-3 / 4} \mathcal{S}_{1}^{n}(g) \stackrel{(d)}{\longrightarrow} N\left(0,\left(t_{1}-t_{0}\right)\left(x_{1}-x_{0}\right)\right) .
$$

Observing that $\|g\|_{2}^{2}=\left(t_{1}-t_{0}\right)\left(x_{1}-x_{0}\right)$, it is easy to see that $\int_{0}^{1} \int g(t, x) W(d t d x)$ has the same distribution. This completes the theorem in this particular case.

Step 2. Still in the $k=1$ case, suppose that $g_{1}, \ldots, g_{m}$ are indicator functions for disjoint, finite area rectangles in $[0,1] \times \mathbb{R}$. Then the variables $\mathcal{S}_{1}^{n}\left(g_{l}\right), l=1, \ldots, m$ are all independent since they are functions of distinct random variables $\omega(i, x)$. Moreover their individual limits $I_{1}\left(g_{l}\right)$ are also independent, since the distinct $g_{l}$ are orthogonal in $L^{2}([0,1] \times \mathbb{R})$. Hence from step 1 and standard theory (see [7], Chapter 1, Section 4) we have the joint convergence

$$
n^{-3 / 4}\left(\mathcal{S}_{1}^{n}\left(g_{1}\right), \ldots, \mathcal{S}_{1}^{n}\left(g_{m}\right)\right) \stackrel{(d)}{\longrightarrow}\left(I_{1}\left(g_{1}\right), \ldots, I_{1}\left(g_{m}\right)\right) .
$$

Step 3. Recall that a vector of variables converges in law if and only if all linear combinations of its entries do (also known as the Cramér-Wold device), so that step 2 implies that for all $\alpha_{1}, \ldots, \alpha_{m} \in \mathbb{R}$,

$$
n^{-3 / 4} \sum_{l=1}^{m} \alpha_{l} \mathcal{S}_{1}^{n}\left(g_{l}\right) \stackrel{(d)}{\longrightarrow} \sum_{l=1}^{m} \alpha_{l} \int_{0}^{1} g_{l}(t, x) W(d t d x) .
$$

However both $\mathcal{S}_{1}^{n}$ and the stochastic integral are linear operators, so

$$
n^{-3 / 4} \mathcal{S}_{1}^{n}\left(\sum_{l=1}^{m} \alpha_{l} g_{l}\right) \stackrel{(d)}{\longrightarrow} \int_{0}^{1} \sum_{l=1}^{m} \alpha_{l} g_{l}(t, x) W(d t d x) .
$$

Hence the theorem holds for all simple functions.

Step 4. In fact joint convergence holds for all finite collections of simple functions. Let $G_{1}, \ldots, G_{M}$ be such a collection, and observe that for any scalars $\beta_{1}, \ldots, \beta_{M} \in \mathbb{R}$ the sum

$$
\sum_{l=1}^{M} \beta_{l} G_{l}
$$

is also a simple function, and hence step 3 applies. By linearity this implies that

$$
\sum_{l=1}^{M} \beta_{l} \mathcal{S}_{1}^{n}\left(g_{l}\right) \stackrel{(d)}{\longrightarrow} \sum_{l=1}^{M} \beta_{l} \int_{0}^{1} G_{l}(t, x) W(d t d x),
$$


and this gives, by the Cramér-Wold device, that

$$
n^{-3 / 4}\left(\mathcal{S}_{1}^{n}\left(G_{1}\right), \ldots, \mathcal{S}_{1}^{n}\left(G_{M}\right)\right) \stackrel{(d)}{\longrightarrow}\left(I_{1}\left(G_{1}\right), \ldots, I_{1}\left(G_{M}\right)\right) .
$$

Step 5. We now complete the proof for $k=1$ via a density argument. For each $g \in L^{2}([0,1] \times \mathbb{R})$ there exists a sequence of simple functions $\left\{g_{N}\right\}_{N \geq 1}$ such that $g_{N} \rightarrow g$ in $L^{2}$, as $N \rightarrow \infty$. Step 3 gives us that

$$
n^{-3 / 4} \mathcal{S}_{1}^{n}\left(g_{N}\right) \stackrel{(d)}{\longrightarrow} \int_{0}^{1} \int g_{N}(t, x) W(d t d x)
$$

as $n \rightarrow \infty$. By $g_{N} \rightarrow g$ in $L^{2}$ we have

$$
\int_{0}^{1} \int g_{N}(t, x) W(d t d x) \stackrel{L^{2}}{\longrightarrow} \int_{0}^{1} \int g(t, x) W(d t d x) .
$$

Similarly, by Lemma 4.1 we have that

$$
Q\left[\left(n^{-3 / 4}\left(\mathcal{S}_{1}^{n}\left(g_{N}\right)-\mathcal{S}_{1}^{n}(g)\right)\right)^{2}\right] \leq\left\|g_{N}-g\right\|_{L^{2}}^{2} .
$$

This implies that, as $N \rightarrow \infty$, the left-hand side converges to zero in $L^{2}$, uniformly in $n$. These three facts combine with Lemma 4.2 to give us the following commutative diagram:

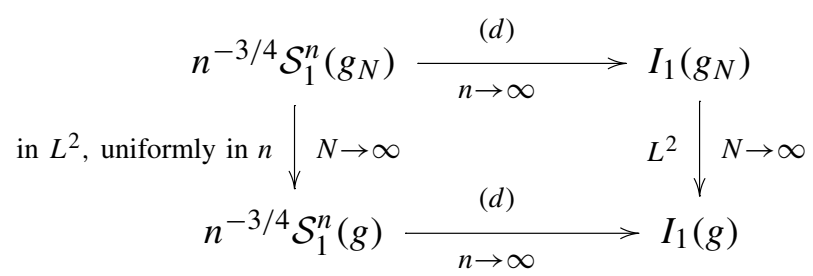

The joint convergence of any finite collection of $n^{-3 / 4} \mathcal{S}_{1}^{n}(g)$ for $g \in L^{2}$ is now a consequence of the above, along with linearity of $\mathcal{S}_{1}^{n}$ and the Cramér-Wold device, as in step 4.

Step 6. Finally, we extend the theorem to $k>1$. First consider functions of the form

$$
g(\mathbf{t}, \mathbf{x})=g_{1}\left(\mathbf{t}_{1}, \mathbf{x}_{1}\right) \cdots g_{k}\left(\mathbf{t}_{k}, \mathbf{x}_{k}\right),
$$

with $g_{j} \in L^{2}([0,1] \times \mathbb{R})$ and distinct $g_{j}$ having distinct support. For such functions $g$,

$$
\begin{aligned}
n^{-k / 2} \mathcal{S}_{k}^{n}(g) & =2^{k / 2} n^{-k / 2} \sum_{\mathbf{i} \in E_{k}^{n}} \sum_{\mathbf{x} \in \mathbb{Z}^{k}} \prod_{j=1}^{k} g_{j}\left(\frac{\mathbf{i}_{j}}{n}, \frac{\mathbf{x}_{j}}{\sqrt{n}}\right) \mathbf{1}\{\mathbf{i} \leftrightarrow \mathbf{x}\} \omega\left(\mathbf{i}_{j}, \mathbf{x}_{j}\right) \\
& =\prod_{j=1}^{k} 2^{1 / 2} n^{-1 / 2} \sum_{i=1}^{n} \sum_{x \in \mathbb{Z}} g_{j}\left(\frac{i}{n}, \frac{x}{\sqrt{n}}\right) \mathbf{1}\{i \leftrightarrow x\} \omega(i, x)
\end{aligned}
$$




$$
\begin{aligned}
& =\prod_{j=1}^{k} n^{-1 / 2} \mathcal{S}_{1}^{n}\left(g_{j}\right) \\
& \stackrel{(d)}{\longrightarrow} \prod_{j=1}^{k} \int_{0}^{1} g_{j}(t, x) W(d t d x) \\
& =\int_{[0,1]^{k}} \int_{\mathbb{R}^{k}} g(\mathbf{t}, \mathbf{x}) W(d \mathbf{t} d \mathbf{x}) .
\end{aligned}
$$

The second and last equalities use that the $g_{j}$ have distinct supports and (27). This proves the result for this particular class of functions, and exactly as in step 4 the result extends to the joint convergence for multiple $g$ of this form, possibly with different $k$. It only remains to finish the proof for general $g \in L^{2}\left([0,1]^{k} \times\right.$ $\mathbb{R}^{k}$ ), which is accomplished via a density argument as in step 5. Functions of the type (30) are dense in $L^{2}\left([0,1]^{k} \times \mathbb{R}^{k}\right)$, and hence step 5 goes through with only trivial modifications.

REMARK. It is worth noting that Theorem 4.3 does not require $g$ to be symmetric even though the stochastic integrals $I_{k}(g)$ are truly only defined for symmetric functions. This is because the operators $\mathcal{S}_{k}^{n}$ have a natural symmetrizing property already built in. If $\pi \in S_{k}$, then it is easy to see that $\overline{(g \circ \pi)}_{n}(\mathbf{t}, \mathbf{x})=$ $\bar{g}_{n}(\pi \mathbf{t}, \pi \mathbf{x})$, where $g \circ \pi(\mathbf{t}, \mathbf{x})=g(\pi \mathbf{t}, \pi \mathbf{x})$, and therefore

$$
\begin{aligned}
\mathcal{S}_{k}^{n}(g \circ \pi) & =2^{k / 2} \sum_{\mathbf{i} \in E_{k}^{n}} \sum_{\mathbf{x} \in \mathbb{Z}^{k}} \bar{g}_{n}\left(\frac{\pi \mathbf{i}}{n}, \frac{\pi \mathbf{x}}{\sqrt{n}}\right) \mathbf{1}\{\mathbf{i} \leftrightarrow \mathbf{x}\} \omega(\mathbf{i}, \mathbf{x}) \\
& =2^{k / 2} \sum_{\mathbf{i} \in E_{k}^{n}} \sum_{\mathbf{x} \in \mathbb{Z}^{k}} \bar{g}_{n}\left(\frac{\mathbf{i}}{n}, \frac{\mathbf{x}}{\sqrt{n}}\right) \mathbf{1}\left\{\pi^{-1} \mathbf{i} \leftrightarrow \pi^{-1} \mathbf{x}\right\} \omega\left(\pi^{-1} \mathbf{i}, \pi^{-1} \mathbf{x}\right)=\mathcal{S}_{k}^{n}(g),
\end{aligned}
$$

the last equality using that $\mathbf{1}\{\mathbf{i} \leftrightarrow \mathbf{x}\} \omega(\mathbf{i}, \mathbf{x})$ is symmetric in its arguments. Hence $\mathcal{S}_{k}^{n}(g)=\mathcal{S}_{k}^{n}(\operatorname{Sym} g)$.

REMARK. If the weight function $g$ is only defined on $\Delta_{k} \times \mathbb{R}^{k}$, then we extend it to $[0,1]^{k} \times \mathbb{R}^{k}$ by setting it to be zero for $\mathbf{t} \notin \Delta_{k}$, as in the remark at the end of Section 3.2. As Sym $g$ is then just a copy of $g$ on each of the $k$ ! permutations of $[0,1]^{k}$, we see that the summation over $E_{k}^{n}$ may be replaced with a summation over $D_{k}^{n}$, with an extra $k$ ! for the proper accounting,

$$
\mathcal{S}_{k}^{n *}(g):=2^{k / 2} \sum_{\mathbf{i} \in D_{k}^{n}} \sum_{\mathbf{x} \in \mathbb{Z}_{k}^{n}} \bar{g}_{n}\left(\frac{\mathbf{i}}{n}, \frac{\mathbf{x}}{\sqrt{n}}\right) \mathbf{1}\{\mathbf{i} \leftrightarrow \mathbf{x}\} \omega(\mathbf{i}, \mathbf{x})=k ! \mathcal{S}_{k}^{n}(g) .
$$

Again by the remark of Section 3.2 this means that

$$
n^{-3 k / 4} \mathcal{S}_{k}^{n *}(g) \stackrel{(d)}{\longrightarrow} \int_{\Delta_{k}} \int_{\mathbb{R}^{k}} g(\mathbf{t}, \mathbf{x}) W(d \mathbf{t} d \mathbf{x}) .
$$


In the future we will drop the $*$ notation on $\mathcal{S}_{k}^{n}$ as it is usually understood from the context what the domain of $g$ is.

Finally we state a theorem which summarizes exactly how discrete Wiener chaos expansions converge to continuum ones in the $L^{2}$ sense.

LEMMA 4.4. If $g=\left(g_{0}, g_{1}, g_{2}, \ldots\right) \in \bigoplus_{k \geq 0} L^{2}\left([0,1]^{k} \times \mathbb{R}^{k}\right)$, then as $n \rightarrow \infty$

$$
I^{n}(g):=\sum_{k=0}^{\infty} n^{-3 k / 4} \mathcal{S}_{k}^{n}\left(g_{k}\right) \stackrel{(d)}{\longrightarrow} \sum_{k=0}^{\infty} \int_{[0,1]^{k}} \int_{\mathbb{R}^{k}} g_{k}(\mathbf{t}, \mathbf{x}) W^{\otimes k}(d \mathbf{t} d \mathbf{x})=I(g)
$$

Moreover if $G_{1}, \ldots, G_{m} \in \bigoplus_{k \geq 0} L^{2}\left([0,1]^{k} \times \mathbb{R}^{k}\right)$, then as $n \rightarrow \infty$ we have the joint convergence

$$
\left(I^{n}\left(G_{1}\right), \ldots, I^{n}\left(G_{m}\right)\right) \stackrel{(d)}{\longrightarrow}\left(I\left(G_{1}\right), \ldots, I\left(G_{m}\right)\right) .
$$

Proof. Since $I$ is an isometry from the Fock space onto $L^{2}\left(\Omega_{W}, \mathcal{F}_{W}, \mathbb{Q}\right)$, we automatically get that $\sum_{k=0}^{M} I_{k}\left(g_{k}\right) \rightarrow \sum_{k=0}^{\infty} I_{k}\left(g_{k}\right)$ in $L^{2}\left(\Omega_{W}, \mathcal{F}_{W}, \mathbb{Q}\right)$, as $M \rightarrow$ $\infty$. Since $\operatorname{Var}\left(n^{-3 k / 4} \mathcal{S}_{k}^{n}\left(g_{k}\right)\right) \leq \operatorname{Var}\left(I_{k}\left(g_{k}\right)\right)$, this also implies that

$$
\sum_{k=0}^{M} n^{-3 k / 4} \mathcal{S}_{k}^{n}\left(g_{k}\right) \stackrel{M \rightarrow \infty}{\longrightarrow} \sum_{k=0}^{\infty} n^{-3 k / 4} \mathcal{S}_{k}^{n}\left(g_{k}\right)
$$

in $L^{2}(\Omega, Q)$, uniformly in $n$. Theorem 4.3 implies that

$$
\sum_{k=0}^{M} n^{-3 k / 4} \mathcal{S}_{k}^{n}\left(g_{k}\right) \stackrel{(d)}{\longrightarrow} \sum_{k=0}^{M} I_{k}\left(g_{k}\right)
$$

as $n \rightarrow \infty$. Putting these pieces together and using Lemma 4.2 gives us the diagram

$$
\begin{aligned}
& \sum_{k=0}^{M} n^{-3 k / 4} \mathcal{S}_{k}^{n}\left(g_{k}\right) \underset{n \rightarrow \infty}{\stackrel{(d)}{\longrightarrow}} \sum_{k=0}^{M} I_{k}\left(g_{k}\right) \\
& \text { uniformly in } L^{2} \downarrow M \rightarrow \infty \quad L^{2} \downarrow M \rightarrow \infty \\
& \sum_{k=0}^{\infty} n^{-3 k / 4} \mathcal{S}_{k}^{n}\left(g_{k}\right) \stackrel{(d)}{n \rightarrow \infty} \longrightarrow \sum_{k=0}^{\infty} I_{k}\left(g_{k}\right) .
\end{aligned}
$$

The joint convergence follows by another application of the Cramér-Wold device. 
4.2. Perturbations of the environment field. Our strategy for proving Theorem 2.1 will be to first prove the convergence result for $\mathfrak{Z}_{n}^{\omega}$, and then extend it to $Z_{n}^{\omega}$. The main idea is that, after a proper deterministic normalization, $Z_{n}^{\omega}$ can be written in the same form as $\mathfrak{Z}_{n}^{\omega}$, but with a mean and variance that may only be asymptotically zero and one, respectively. In this section we give sufficient conditions on the mean and variance so that Theorem 4.3 still holds.

Throughout we let $\tilde{\omega}_{n}(i, x)$ denote a field of i.i.d. random variables on $\mathbb{N} \times$ $\mathbb{Z}$. The dependence on $n$ is to indicate that the distribution of the environment variables may vary with $n$. For $g \in L^{2}\left([0,1]^{k} \times \mathbb{R}^{k}\right)$ we let $\mathcal{S}_{k}^{n}\left(g ; \tilde{\omega}_{n}\right)$ denote the same quantity as $\mathcal{S}_{k}^{n}(g)$, but with the $\omega$ variables replaced by $\tilde{\omega}_{n}$. We have the following generalization of Theorem 4.3:

THEOREM 4.5. Assume that the environment variables $\tilde{\omega}_{n}$ satisfy $Q\left(\tilde{\omega}_{n}\right)=0$ and $Q\left(\tilde{\omega}_{n}^{2}\right)=1+o(1)$. Then the statement of Theorem 4.3 holds with all instances of $\mathcal{S}_{k}^{n}(g)$ replaced by $\mathcal{S}_{k}^{n}\left(g ; \tilde{\omega}_{n}\right)$.

PROOF. Re-examining the proof of Theorem 4.3, we see that it is enough to check that steps 1 and 5 are still valid; the other steps are unchanged. For step 1 we again assume that $g(t, x)=\mathbf{1}\left\{t_{0}<t \leq t_{1}, x_{0}<x \leq x_{1}\right\}$, and we want to show that $n^{-3 / 4} \mathcal{S}_{1}^{n}\left(g ; \tilde{\omega}_{n}\right)$ converges in law to a Gaussian with mean zero and variance $\|g\|_{2}^{2}$. Since the distribution of the random variables is allowed to change with $n$ we require extra hypotheses found in the central limit theorems for triangular arrays. By [20], Theorem 2.4.5, the given hypothesis on the mean and variance are sufficient. The cited theorem also requires a condition of the form $Q\left(\tilde{\omega}_{n}^{2} \mathbf{1}\left\{\left|\tilde{\omega}_{n}\right|>\right.\right.$ $\varepsilon \sqrt{n}\}) \rightarrow 0$ for every $\varepsilon>0$, but since the $\tilde{\omega}_{n}$ variables are i.i.d. (for each $n$ ) the hypothesis on the variance covers this; see the remark after [20], Theorem 2.4.5.

For step 5 observe that we now have

$$
Q\left[\prod_{j=1}^{k} \tilde{\omega}_{n}\left(\mathbf{i}_{j}, \mathbf{x}_{j}\right) \prod_{j=1}^{k} \tilde{\omega}_{n}\left(\mathbf{i}_{j}^{\prime}, \mathbf{x}_{j}^{\prime}\right)\right]=(1+o(1))^{k} \mathbf{1}\left\{\mathbf{i}=\mathbf{i}^{\prime}, \mathbf{x}=\mathbf{x}^{\prime}\right\} .
$$

Correspondingly, we have as an analogue of Lemma 4.1

$$
Q\left(\mathcal{S}_{k}^{n}\left(g ; \tilde{\omega}_{n}\right)^{2}\right) \leq n^{3 k / 2}(1+o(1))^{k} Q\left(\tilde{\omega}_{n}^{2}\right)^{k}\|g\|_{L^{2}\left([0,1]^{k} \times \mathbb{R}^{k}\right)}^{2} .
$$

Now for $g \in L^{2}([0,1] \times \mathbb{R})$ again let $g_{N}$ be a sequence of simple functions such that $g_{N} \rightarrow g$ in $L^{2}$. All that needs to be checked is that $n^{-3 / 4} \mathcal{S}_{1}^{n}\left(g_{N} ; \tilde{\omega}_{n}\right) \rightarrow$ $n^{-3 / 4} \mathcal{S}_{1}^{n}\left(g ; \tilde{\omega}_{n}\right)$ in $L^{2}$, uniformly in $n$, but this is obvious since by the last calculation,

$$
Q\left(\left(n^{-3 / 4} \mathcal{S}_{1}^{n}\left(g_{N}-g ; \tilde{\omega}_{n}\right)\right)^{2}\right) \leq(1+o(1))\left\|g_{N}-g\right\|_{L^{2}}^{2}
$$

We now extend Lemma 4.4 to the situation of the previous lemma. 
LEMMA 4.6. Let $\tilde{\omega}_{n}$ satisfy the hypothesis of the previous theorem, and assume that $g=\left(g_{0}, g_{1}, \ldots,\right) \in \bigoplus_{k \geq 0} L^{2}\left([0,1]^{k} \times \mathbb{R}^{k}\right)$ is such that

$$
\lim _{N \rightarrow \infty} \limsup _{n \rightarrow \infty} \sum_{k=N}^{\infty} Q\left(\tilde{\omega}_{n}^{2}\right)^{k}\left\|g_{k}\right\|_{L^{2}}^{2}=0,
$$

then

$$
\tilde{I}^{n}(g):=\sum_{k=0}^{\infty} n^{-3 k / 4} \mathcal{S}_{k}^{n}\left(g_{k} ; \tilde{\omega}_{n}\right) \stackrel{(d)}{\longrightarrow} I(g)
$$

as $n \rightarrow \infty$. Moreover if $G_{1}, \ldots, G_{M} \in \bigoplus L^{2}\left([0,1]^{k} \times \mathbb{R}^{k}\right)$ individually satisfy (31), then

$$
\left(\tilde{I}^{n}\left(G_{1}\right), \ldots, \tilde{I}^{n}\left(G_{M}\right)\right) \stackrel{(d)}{\longrightarrow}\left(I\left(G_{1}\right), \ldots, I\left(G_{M}\right)\right) .
$$

PROOF. The proof of Lemma 4.4 goes through as before; one only needs to check that

$$
\sum_{k=0}^{M} n^{-3 k / 4} \mathcal{S}_{k}^{n}\left(g_{k} ; \tilde{\omega}_{n}\right) \rightarrow \sum_{k=0}^{\infty} n^{-3 k / 4} \mathcal{S}_{k}^{n}\left(g_{k} ; \tilde{\omega}_{n}\right)
$$

uniformly in $n$ as $M \rightarrow \infty$, and (31) guarantees that this is true in the $L^{2}$ sense. The joint convergence again follows from the Cramér-Wold device: one only needs to check that condition (31) is satisfied by any linear combination of the $G_{i}$, and this is an easy consequence of the triangle inequality.

REMARK. The assumption that $Q\left(\tilde{\omega}_{n}\right)=0$ is not really necessary; in general $Q\left(\tilde{\omega}_{n}\right)=o\left(n^{-3 / 4}\right)$ will suffice. However, in this case the cross product terms take the form

$$
\begin{aligned}
& Q\left[\prod_{j=1}^{k} \tilde{\omega}_{n}\left(\mathbf{i}_{j}, \mathbf{x}_{j}\right) \prod_{j=1}^{k} \tilde{\omega}_{n}\left(\mathbf{i}_{j}^{\prime}, \mathbf{x}_{j}^{\prime}\right)\right] \\
& \quad=Q\left(\tilde{\omega}_{n}^{2}\right)^{\#\left\{j:\left(\mathbf{i}_{j}, \mathbf{x}_{j}\right)=\left(\mathbf{i}_{j}^{\prime}, \mathbf{x}_{j}^{\prime}\right)\right\}} Q\left(\tilde{\omega}_{n}\right)^{2 \#\left\{j:\left(\mathbf{i}_{j}, \mathbf{x}_{j}\right) \neq\left(\mathbf{i}_{j}^{\prime}, \mathbf{x}_{j}^{\prime}\right)\right\}},
\end{aligned}
$$

which is cumbersome to deal with.

5. Convergence of the point-to-line partition functions. In this section we use the results of Section 4 to prove Theorem 2.1 on convergence of the partition functions. The strategy is to prove the convergence for the modified partition functions $\mathfrak{Z}_{n}^{\omega}$ and then transfer the results to the usual partition functions $Z_{n}^{\omega}$. Throughout we assume that the environment variables have mean zero and variance one.

DEFINITION 5.1. For $k, n \geq 1$, define $p_{k}^{n}:[0,1]^{k} \times \mathbb{R}^{k} \rightarrow \mathbb{R}$ by

$$
p_{k}^{n}(\mathbf{t}, \mathbf{x})=\bar{p}_{k}(\lceil n \mathbf{t}\rceil, \mathbf{x} \sqrt{n}) \mathbf{1}\left\{\lceil n \mathbf{t}\rceil \in D_{k}^{n}\right\} .
$$


REMARK. Observe that for $k>n$, it is impossible for a vector in $[0,1]^{k}$ to have all $k$ elements separated by at least $1 / n$. Hence the condition $\lceil n \mathbf{t}\rceil \in D_{k}^{n}$ implies that $p_{k}^{n}$ is identically zero.

REMARK. Temporarily ignoring the indicator function term in this last definition, $p_{k}^{n}$ is the $k$-fold density function of the continuous time process

$$
t \mapsto \frac{\mathcal{S}_{\lceil n t\rceil}+U_{\lceil n t\rceil}}{\sqrt{n}},
$$

where the $U_{i}$ are an i.i.d. collection of Uniform random variables on $(-1,1)$. We will use this later to simplify definitions for the point-to-point partition functions.

REMARK. Also observe that $p_{k}^{n}$ is already constant on the rectangles of $\mathcal{R}_{k}^{n}$, so that $\overline{p_{k}^{n}}=p_{k}^{n}$. Moreover, for $\mathbf{i} \in \mathrm{E}_{k}^{n}, \mathbf{x} \in \mathbb{Z}^{k}$ such that $\mathbf{i} \leftrightarrow \mathbf{x}$,

$$
p_{k}^{n}\left(\frac{\mathbf{i}}{n}, \frac{\mathbf{x}}{\sqrt{n}}\right)=\bar{p}_{k}(\mathbf{i}, \mathbf{x}) \mathbf{1}\left\{\mathbf{i} \in D_{k}^{n}\right\}=2^{-k} p_{k}(\mathbf{i}, \mathbf{x}) \mathbf{1}\left\{\mathbf{i} \in D_{k}^{n}\right\} .
$$

Thus, by definition (29) of $\mathcal{S}_{k}^{n}$,

$$
\mathcal{S}_{k}^{n}\left(p_{k}^{n}\right)=2^{-k / 2} \sum_{\mathbf{i} \in D_{k}^{n}} \sum_{\mathbf{x} \in \mathbb{Z}^{k}} p_{k}(\mathbf{i}, \mathbf{x}) \omega(\mathbf{i}, \mathbf{x}) .
$$

The $\mathbf{i} \leftrightarrow \mathbf{x}$ condition is already handled by $p_{k}$. This leads to the following:

LEMMA 5.2. The point-to-line partition function may be rewritten as

$$
\mathfrak{Z}_{n}^{\omega}(\beta)=\sum_{k=0}^{n} 2^{k / 2} \beta^{k} \mathcal{S}_{k}^{n}\left(p_{k}^{n}\right)=\sum_{k=0}^{n} 2^{k / 2} \beta^{k} n^{-k / 2} \mathcal{S}_{k}^{n}\left(n^{k / 2} p_{k}^{n}\right) .
$$

Consequently,

$$
\mathfrak{Z}_{n}^{\omega}\left(\beta n^{-1 / 4}\right)=\sum_{k=0}^{n} 2^{k / 2} \beta^{k} n^{-3 k / 4} \mathcal{S}_{k}^{n}\left(n^{k / 2} p_{k}^{n}\right)
$$

From this we have the following:

PROPOSITION 5.3. Assume that the environment variables $\omega$ have mean zero and variance one. Then as $n \rightarrow \infty$ we have $\mathfrak{Z}_{n}^{\omega}\left(\beta n^{-1 / 4}\right) \stackrel{(d)}{\longrightarrow} \mathcal{Z}_{\sqrt{2} \beta}$.

PROOF. First observe that Lemma 4.4 implies that for all $\beta>0$,

$$
\sum_{k=0}^{\infty} \beta^{k} n^{-3 k / 4} \mathcal{S}_{k}^{n}\left(\varrho_{k}\right) \stackrel{(d)}{\longrightarrow} \mathcal{Z}_{\beta}
$$


as $n \rightarrow \infty$. Now we show that the difference between this term and $\mathfrak{Z}_{n}^{\omega}\left(\beta n^{-1 / 4}\right)$ goes to zero as $n \rightarrow \infty$. Observe that

$$
\begin{aligned}
& \sum_{k=0}^{\infty} 2^{k / 2} \beta^{k} n^{-3 k / 4} \mathcal{S}_{k}^{n}\left(\varrho_{k}\right)-\mathfrak{Z}_{n}^{\omega}\left(\beta n^{-1 / 4}\right) \\
& \quad=\sum_{k=0}^{n} 2^{k / 2} \beta^{k} n^{-3 k / 4} \mathcal{S}_{k}^{n}\left(\varrho_{k}-n^{k / 2} p_{k}^{n}\right)+\sum_{k=n+1} 2^{k / 2} \beta^{k} n^{-3 k / 4} \mathcal{S}_{k}^{n}\left(\varrho_{k}\right) .
\end{aligned}
$$

By Lemma 4.1 the second term is bounded in $L^{2}$ by

$$
\sum_{k=n+1}^{\infty} 2^{k} \beta^{2 k}\left\|\varrho_{k}\right\|_{L^{2}\left([0,1]^{k} \times \mathbb{R}^{k}\right)}^{2},
$$

which, by the estimates in Section 3.4, goes to zero as $n \rightarrow \infty$. For the first term, note that in $L^{2}$ it is bounded above by

$$
\sum_{k=0}^{n} 2^{k} \beta^{2 k}\left\|\varrho_{k}-n^{k / 2} p_{k}^{n}\right\|_{L^{2}}^{2}
$$

The local limit theorem implies that $n^{k / 2} p_{k}^{n} \rightarrow \varrho_{k}$ pointwise as $n \rightarrow \infty$. In Lemma A. 1 of the Appendix we prove there is a constant $C>0$ such that

$$
\sup _{n}\left\|n^{k / 2} p_{k}^{n}\right\|_{L^{2}} \leq C^{k}\left\|\varrho_{k}\right\|_{L^{2}}
$$

hence by the triangle inequality and dominated convergence we have $\| \varrho_{k}-$ $n^{k / 2} p_{k}^{n} \|_{L^{2}} \rightarrow 0$ as $n \rightarrow \infty$. Since the sequence $C^{k}\left\|\varrho_{k}\right\|_{L^{2}}$ is summable, the estimate above and dominated convergence also imply that

$$
\lim _{n \rightarrow \infty} \sum_{k=0}^{n} 2^{k} \beta^{2 k}\left\|\varrho_{k}-n^{k / 2} p_{k}^{n}\right\|_{L^{2}}^{2}=\sum_{k=0}^{\infty} 2^{k} \beta^{2 k} \lim _{n \rightarrow \infty}\left\|\varrho_{k}-n^{k / 2} p_{k}^{n}\right\|_{L^{2}}^{2}=0 .
$$

Now we begin the process of extending the convergence to the partition function $Z_{n}^{\omega}$.

Proposition 5.4. Suppose that there is a $\beta_{0}>0$ such that $\lambda(\beta):=$ $\log Q\left(e^{\beta \omega}\right)<\infty$ for all $0<\beta<\beta_{0}$. Then

$$
e^{-n \lambda\left(\beta n^{-1 / 4}\right)} Z_{n}^{\omega}\left(\beta n^{-1 / 4}\right) \stackrel{(d)}{\longrightarrow} \mathcal{Z}_{\sqrt{2} \beta} .
$$

ProOF. Define the environment field $\tilde{\omega}_{n}$ by

$$
e^{\beta n^{-1 / 4} \omega(i, x)-\lambda\left(\beta n^{-1 / 4}\right)}=1+\beta n^{-1 / 4} \tilde{\omega}_{n}(i, x),
$$


so that

$$
\begin{aligned}
Z_{n}^{\omega}\left(\beta n^{-1 / 4}\right) & =\mathbf{P}\left[\prod_{i=1}^{n}\left(1+\beta n^{-1 / 4} \tilde{\omega}_{n}\left(i, S_{i}\right)\right)\right] \\
& =\mathfrak{Z}_{n}^{\tilde{\omega}_{n}}\left(\beta n^{-1 / 4}\right) .
\end{aligned}
$$

It is straightforward to check that exponential moments for $\omega$ and the definition of $\lambda(\beta)$ imply that $\tilde{\omega}_{n}$ satisfy $Q\left(\tilde{\omega}_{n}\right)=0$ and $Q\left(\tilde{\omega}_{n}^{2}\right)=1+O\left(n^{-1 / 4}\right)$, which are even stronger than the hypotheses of Theorem 4.5. The proof is now completed by using Lemma 4.6 and mimicking the proof of Proposition 5.3; briefly, it follows that

$$
\sum_{k=0}^{\infty} \beta^{k} n^{-3 k / 4} \mathcal{S}_{k}^{n}\left(\varrho_{k} ; \tilde{\omega}_{n}\right) \stackrel{(d)}{\longrightarrow} \mathcal{Z}_{\beta}
$$

as $n \rightarrow \infty$. This is clear by Lemma 4.6 since $C^{k}\left\|\varrho_{k}\right\|_{L^{2}}$ is summable in $k$ for any $C>0$. Furthermore

$$
\sum_{k=0}^{\infty} 2^{k / 2} \beta^{k} \mathcal{S}_{k}^{n}\left(\varrho_{k}-n^{k / 2} p_{k}^{n} ; \tilde{\omega}_{n}\right)
$$

also goes to zero for the same reason as in Proposition 5.3, by using Lemma A.1 as before.

6. Convergence of the point-to-point partition functions. In this section we describe the proofs behind Theorems 2.2 and 2.7. The arguments are based on the ones for the point-to-line partition function.

6.1. The random local limit theorem. We extend the methods of the last section to prove convergence of the endpoint density under intermediate disorder. We will prove all parts of Theorem 2.2 except for the tightness, the proof of which is delayed until the Appendix. Much of what we describe in this section is a relatively simple extension of the convergence of the point-to-line partition function so we do not go into as much detail.

Appealing to equation (12), we easily see that the term

$$
\mathfrak{Z}_{n}^{\omega}\left(x \sqrt{n} ; \beta n^{-1 / 4}\right)
$$

may be written as the sum of $n$ terms in a discrete Wiener chaos expansion, in an analogous way to what was done in Lemma 5.2. Define $p_{k \mid x}^{n}$ on $[0,1]^{k} \times \mathbb{R}^{k}$ by

$$
n^{k / 2} p_{k \mid x}^{n}(\mathbf{t}, \mathbf{x})=\mathbf{P}\left(X_{\mathbf{t}_{1}} \in d \mathbf{x}_{1}, \ldots, X_{\mathbf{t}_{k}} \in d \mathbf{x}_{k} \mid X_{1} \in d x\right) \mathbf{1}\left\{\lceil n \mathbf{t}\rceil \in D_{k}^{n}\right\},
$$


where $X_{t}=\left(S_{\lceil n t\rceil}+U_{\lceil n t\rceil}\right) / \sqrt{n}$ is the continuous time process as defined before in Section 2.1. Using this, we may rewrite (12) as

$$
\begin{gathered}
\mathbf{P}\left[\prod_{i=1}^{n}\left(1+\beta n^{-1 / 4} \omega\left(i, S_{i}\right)\right) \mid S_{n}=x \sqrt{n}\right] \\
=\sum_{k=0}^{n} 2^{k / 2} \beta^{k} n^{-3 k / 4} \mathcal{S}_{k}^{n}\left(n^{k / 2} p_{k \mid x}^{n}\right) .
\end{gathered}
$$

On the right-hand side the $\sqrt{n}$ spatial scaling on $x$ has already been factored into the definition of $p_{k \mid x}^{n}$. On the other hand, defining $\varrho_{k \mid x}$ to be the $k$-fold density of a Brownian bridge from 0 to $x$, that is,

$$
\varrho_{k \mid x}(\mathbf{t}, \mathbf{x})=\frac{\varrho\left(1-\mathbf{t}_{k}, x-\mathbf{x}_{k}\right)}{\varrho(1, x)} \varrho_{k}(\mathbf{t}, \mathbf{x}),
$$

it follows immediately from Lemma 4.4 that

$$
\left\{x \mapsto \sum_{k=0}^{\infty} n^{-3 k / 4} \mathcal{S}_{k}^{n}\left(\varrho_{k \mid x}\right)\right\} \stackrel{(d)}{\longrightarrow}\left\{x \mapsto e^{A_{\beta}(x)}\right\}
$$

as $n \rightarrow \infty$, in the sense of convergence of finite dimensional distributions; see (14) for the definition of $A_{\beta}(x)$. All that remains to be shown is that the difference between this process and

$$
x \mapsto \sum_{k=0}^{n} n^{-3 k / 4} \mathcal{S}_{k}^{n}\left(n^{k / 2} p_{k \mid x}^{n}\right)
$$

goes to zero as $n \rightarrow \infty$. The difference breaks into two terms, the easiest of which to deal with is

$$
\sum_{k=n+1}^{\infty} 2^{k / 2} \beta^{k} n^{-3 k / 4} \mathcal{S}_{k}^{n}\left(\varrho_{k \mid x}\right) .
$$

By Lemma 4.1 and the comments at the end of Section 3.4 this term goes to zero in $L^{2}$ at a rate that is uniform in $x$. The other term to deal with is

$$
\sum_{k=0}^{n} 2^{k / 2} \beta^{k} n^{-3 k / 4} \mathcal{S}_{k}^{n}\left(\varrho_{k \mid x}-n^{k / 2} p_{k \mid x}^{n}\right),
$$

which has variance bounded above by

$$
\sum_{k=0}^{n} 2^{k} \beta^{2 k}\left\|\varrho_{k \mid x}-n^{k / 2} p_{k \mid x}^{n}\right\|_{L^{2}}^{2}
$$

To show this latter term goes to zero requires another application of dominated convergence as in the last section, and this uses the estimates of Lemma A.1. 
To move from convergence of (32) to convergence of $\mathfrak{Z}_{n}^{\omega}\left(x \sqrt{n} ; \beta n^{-1 / 4}\right)$ is a simple matter since the two differ by only a factor of $p(n, x \sqrt{n})$. The local limit theorem immediately implies that $\sqrt{n} p(n, x \sqrt{n}) / 2$ converges to $\rho(1, x)$ as $n \rightarrow$ $\infty$, and modulo the tightness this completes the proof of (15) that

$$
\left\{x \mapsto \frac{\sqrt{n}}{2} \mathfrak{Z}_{n}^{\omega}\left(x \sqrt{n} ; \beta n^{-1 / 4}\right)\right\} \stackrel{(d)}{\longrightarrow}\left\{x \mapsto e^{A_{\sqrt{2} \beta}(x)} \varrho(1, x)\right\} .
$$

Since Lemma 4.4 also implies joint convergence of the point-to-line partition function and the point-to-point partition function at any finite collection of points, this implies the random local limit theorem for the endpoint density

$$
\left\{x \mapsto \frac{\sqrt{n} \mathfrak{Z}_{n}^{\omega}\left(x \sqrt{n} ; \beta n^{-1 / 4}\right) / 2}{\mathfrak{Z}_{n}^{\omega}\left(\beta n^{-1 / 4}\right)}\right\} \stackrel{(d)}{\longrightarrow}\left\{x \mapsto \frac{e^{A_{\beta}(x)} \varrho(1, x)}{\mathcal{Z}_{\beta}}\right\} .
$$

Finally it only remains to extend the result for the $\mathfrak{Z}_{n}^{\omega}$ partition functions to the $Z_{n}^{\omega}$ ones. This is accomplished the same way as in the last section by introducing the $\tilde{\omega}_{n}$ environment field and using the relation $Z_{n}^{\omega}=\mathfrak{Z}_{n}^{\tilde{\omega}_{n}}$. The only extra work required is in showing that the conditions of Lemma 4.6 are satisfied, but, as for the point-to-line partition function, this is an easy consequence of the estimates in the Appendix.

6.2. Convergence of the four-parameter field. Now we prove convergence of the four-parameter field of transition probabilities as stated in Theorem 2.7. As in the last section we defer the tightness until the Appendix and concentrate on convergence of the finite dimensional distributions. This follows the same scheme as before. Partition functions of the form

$$
\mathfrak{Z}^{\omega}(m, y ; k, x ; \beta)
$$

[see (24) for the definition] are point-to-point partition functions shifted to a different starting point. Using the techniques of the last section we know that its law converges under intermediate disorder scaling for the environment if space and time are scaled diffusively, and hence this implies that

$$
\frac{\sqrt{n}}{2} \mathfrak{Z}^{\omega}\left(n s, y \sqrt{n} ; n t, x \sqrt{n} ; \beta n^{-1 / 4}\right) \stackrel{(d)}{\longrightarrow} \mathcal{Z}_{\sqrt{2} \beta}(s, y ; t, x)
$$

as $n \rightarrow \infty$. This can also be seen by expanding (24) as a discrete chaos series of type (32), using kernels of the form

$$
\begin{aligned}
& n^{k / 2} p_{k \mid(s, y ; t, x)}^{n}(\mathbf{t}, \mathbf{x}) \\
& \quad:=\mathbf{P}\left(X_{\mathbf{t}_{1}} \in d \mathbf{x}_{1}, \ldots, X_{\mathbf{t}_{k}} \in d \mathbf{x}_{k} \mid X_{s} \in d y, X_{t} \in d x\right) \mathbf{1}\left\{\lceil n t\rceil \in D_{k}^{n}\right\} .
\end{aligned}
$$

These kernels are space-time shifts of the kernels $n^{k / 2} p_{k \mid x}^{n}$. Shift invariance of the underlying random walk implies that these kernels are simple translates of each other. Since shifting the kernels is equivalent to shifting the field of environment 
random variables, and the law of the field is clearly shift invariant, the shifted partition function is equal in law to the unshifted one. More precisely, we have equality in law of

$\frac{\sqrt{n}}{2} \mathfrak{Z}^{\omega}\left(n s, y \sqrt{n} ; n t, x \sqrt{n} ; \beta n^{-1 / 4}\right) \equiv \frac{\sqrt{n}}{2} \mathfrak{Z}^{\omega}\left(0,0 ; n(t-s),(x-y) \sqrt{n} ; \beta n^{-1 / 4}\right)$.

The law of the right-hand side converges by the arguments in Section 6.1, which is just the special case of $s=0$ and $t=1$. For a finite collection of space-time points $\left(s_{i}, y_{i} ; t_{i}, x_{i}\right)$ the joint convergence of

$$
\frac{\sqrt{n}}{2} \mathfrak{Z}^{\omega}\left(n s_{i}, y_{i} \sqrt{n} ; n t_{i}, x_{i} \sqrt{n} ; \beta n^{-1 / 4}\right)
$$

follows by Lemma 4.4.

Similarly, partition functions of the form

$$
\mathfrak{Z}_{n}^{\omega}(m, y ; k, * ; \beta)
$$

are point-to-line partition functions shifted to a different starting point. They can be expanded into discrete Wiener chaos using the kernel functions

$$
n^{k / 2} p_{k \mid(s, y ; t, *)}^{n}(\mathbf{t}, \mathbf{x})=\mathbf{P}\left(X_{\mathbf{t}_{1}} \in d \mathbf{x}_{1}, \ldots, X_{\mathbf{t}_{k}} \in d \mathbf{x}_{k} \mid X_{s} \in d y\right) .
$$

Using the methods of Section 5 their law converges under the intermediate disorder scaling on the environment and diffusive scaling on space and time. Joint convergence of

$$
\mathfrak{Z}^{\omega}\left(n s_{i}, y_{i} \sqrt{n} ; n t_{i}, * ; \beta n^{-1 / 4}\right)
$$

at finitely many points $\left(s_{i}, y_{i} ; t_{i}\right)$ follows from Lemma 4.4 .

To convert convergence of the $\mathfrak{Z}^{\omega}$ partition functions into the $Z^{\omega}$ partition functions, introduce again the field $\tilde{\omega}_{n}$ and use the relation $Z^{\omega}=\mathfrak{Z}^{\tilde{\omega}_{n}}$. For example, it is clear under this definition that

$$
\begin{aligned}
& \mathfrak{Z}^{\tilde{\omega}_{n}}(\left.n s, y \sqrt{n} ; n t, x \sqrt{n} ; \beta n^{-1 / 4}\right) \\
& \quad=e^{-n(t-s) \lambda\left(\beta n^{-1 / 4}\right)} Z^{\omega}\left(n s, y \sqrt{n} ; n t, x \sqrt{n} ; \beta n^{-1 / 4}\right),
\end{aligned}
$$

up to a negligible difference caused by the term $\exp \left\{-n(t-s) \lambda\left(\beta n^{-1 / 4}\right)\right\}$ (since $t-s$ is not usually a multiple of $1 / n)$. The left-hand side converges from the arguments just discussed, and joint convergence at a finite collection of space-time points $\left(s_{i}, y_{i} ; t_{i}, x_{i}\right)$ follows from Lemma 4.6. A similar argument shows convergence of the finite dimensional distributions for the field of $Z^{\omega}$ point-to-line partition functions.

7. Concluding remarks. We end with a few brief observations and ideas for future work. 
7.1. Supercritical scaling. The $n^{-1 / 4}$ scaling on the environment is the hallmark of the intermediate disorder regime. Under this scaling the law of the random polymer measure converges, although with probability one the polymer measure itself does not converge. In this respect the $n^{-1 / 4}$ scaling is sharp, meaning that if one replaces $1 / 4$ by any larger exponent then the resulting polymer would be in the weak disorder regime. More precisely:

SUPERCRITICAL SCALING. Under the scaling $\beta_{n}=\beta n^{-(1 / 4+\delta)}$ for any $\delta>0$ :

- the partition function $e^{-n \lambda\left(\beta_{n}\right)} Z_{n}^{\omega}\left(\beta n^{-(1 / 4+\delta)}\right)$ converges in probability to 1 ;

- the endpoint density, under diffusive scaling of space, converges to the standard Gaussian distribution;

- the transition probabilities converge, under diffusive scaling of space and time, to the transition probabilities for standard Brownian motion.

The idea behind the proof of these results is already apparent in the proofs of Theorems 2.1 and 2.2. The extra term of $n^{-\delta}$ in the exponent sends each of the random terms of (6) and (12) to zero (in $L^{2}$ ), and all that survives is the deterministic first-order terms. These correspond to Gaussian endpoint fluctuations. Full details for the point-to-line partition function are given in [22].

7.2. Subcritical scaling. Replacing the $1 / 4$ exponent by a smaller value produces a regime that we are unable to analyze rigorously but for which we have many conjectures. Consider the scalings $\beta_{n}:=\beta n^{-\alpha}$ for $0 \leq \alpha<1 / 4$. Under this scaling our methodology breaks down because the individual terms of the discrete Wiener chaos blow up as $n \rightarrow \infty$. Our conjectured value for the fluctuation exponents are

$$
\chi(\alpha)=\frac{1}{3}(1-4 \alpha), \quad \zeta(\alpha)=\frac{2}{3}(1-\alpha) .
$$

Observe that these values linearly interpolate between the conjectured values of $\zeta=2 / 3, \chi=1 / 3$ at $\alpha=0$, and the values $\zeta=1 / 2, \chi=0$ that we prove in this paper. Moreover, $\chi(\alpha)$ and $\zeta(\alpha)$ satisfy the relation

$$
\chi(\alpha)=2 \zeta(\alpha)-1,
$$

which is already predicted in the $\alpha=0$ case. Further details of this conjecture (and others) are discussed in [3].

7.3. Assumptions on moments. In this paper we have two different sets of assumptions on the moments of the environment random variables, each set corresponding to a different Hamiltonian used to construct the polymer measure. In the first case, for the partition function $\mathfrak{Z}_{n}^{\omega}$, all of our results on intermediate disorder only require that the environment variables have finite variance for convergence of 
the finite dimensional distributions. This is essentially a consequence of the central limit theorem. However, for the more commonly studied partition function $Z_{n}^{\omega}$, it is clear that more moments are required. In order for the partition function $Z_{n}^{\omega}(\beta)$ to have finite mean it is necessary that the environment variables have finite exponential moments. It is possible, however, that even without this assumption the $Z_{n}^{\omega}$ partition function converges to the same limits as before, and we believe this to be the case. In fact we believe that it is sufficient that the environment variables have more than 6 moments. Loosely speaking, this conjecture is based on the idea that the path measure with enough moments has diffusive scalings under intermediate disorder. This suggests that $n^{3 / 2}$ environment variables are all that is contributing to the partition function, and as long as no single one of these variables is dominant, the convergence will be enforced by the central limit theorem. Since each environment variable is multiplied by a factor of $n^{-1 / 4}$ under intermediate disorder, a simple Chebyshev bound shows that greater than 6 moments is sufficient to keep all of them order one. A similar argument indicates that the condition of 6 moments is sharp, and that partition functions with less than 6 moments do not converge to the universal limits in the intermediate disorder regime. It is interesting to compare this moment condition with the case of the unscaled environment (strong disorder), where it is widely believed [8] that greater than 4 moments is sufficient for convergence to the universal limit governed by Tracy-Widom laws.

7.4. Crossover on the process level. As was explained, under proper scalings the one-point marginal distributions of the process $A_{\beta}(x)$ cross over from a Gaussian distribution to the Tracy-Widom GUE. It is natural then to conjecture that the process converges from a stationary Gaussian process to the Airy 2 process, with proper scalings in the $x$ variable as $\beta$ approaches 0 and $\infty$. It is tempting to try to prove at least the tightness in the limit as $\beta \rightarrow \infty$ using the control provided by the convergent power series for the process $A_{\beta}(x)$. Such hypothetical asymptotic analysis in the limit $\beta \rightarrow \infty$ will not produce an exact expression for the Airy $_{2}$ process, but might shed some light on the critical exponents $2 / 3$ and $1 / 3$, and might help to explain the localization phenomenon for the path measure in the strong disorder regime.

APPENDIX A: BOUNDS ON DISCRETE RANDOM WALK PROBABILITIES

LEMMA A.1. There exists a constant $C>0$ such that for all $k \geq 0$ and $x \in \mathbb{R}$, $\sup _{n}\left\|n^{k / 2} p_{k}^{n}\right\|_{L^{2}} \leq C^{k}\left\|\varrho_{k}\right\|_{L^{2}}, \quad \sup _{n}\left\|n^{k / 2} p_{k \mid x}^{n}\right\|_{L^{2}} \leq C^{k}\left\|\varrho_{k \mid x}\right\|_{L^{2}}$.

PROOF. First observe that there exists a constant $C$ such that $\sqrt{i} p(i, x) \leq C$ for all $i$ and $x$, and therefore

$$
\sup _{\mathbf{x} \in \mathbb{Z}^{k}} p_{k}(\mathbf{i}, \mathbf{x}) \leq C^{k} \prod_{j=1}^{k} \frac{1}{\sqrt{\mathbf{i}_{j}-\mathbf{i}_{j-1}}} .
$$


From this and by definition of $p_{k}^{n}$ we have

$$
\begin{aligned}
\left\|n^{k / 2} p_{k}^{n}\right\|_{L^{2}}^{2} & =n^{k} \sum_{\mathbf{i} \in D_{k}^{n}} \sum_{\mathbf{x} \in \mathbb{Z}^{k}} p_{k}(\mathbf{i}, \mathbf{x})^{2} n^{-3 k / 2} \\
& \leq n^{-k / 2} \sum_{\mathbf{i} \in D_{k}^{n} \mathbf{y} \in \mathbb{Z}^{k}} \max _{k}(\mathbf{i}, \mathbf{y}) \sum_{\mathbf{x} \in \mathbb{Z}^{k}} p_{k}(\mathbf{i}, \mathbf{x}) \\
& \leq C^{k} n^{-k / 2} \sum_{\mathbf{i} \in D_{k}^{n}} \prod_{j=1}^{k} \frac{1}{\sqrt{\mathbf{i}_{j}-\mathbf{i}_{j-1}}} \\
& =C^{k} n^{-k} \sum_{\mathbf{i} \in D_{k}^{n}} \prod_{j=1}^{k}\left(\frac{\mathbf{i}_{j}}{n}-\frac{\mathbf{i}_{j-1}}{n}\right)^{-1 / 2} \\
& \leq C^{k} \int_{\Delta_{k}} \prod_{j=1}^{k} \frac{1}{\sqrt{\mathbf{t}_{j}-\mathbf{t}_{j-1}}} d \mathbf{t} \\
& \leq\left(C^{\prime}\right)^{k}\left\|\varrho_{k}\right\|_{L^{2}}^{2} .
\end{aligned}
$$

The second-to-last inequality between the sum and the integral is an easy consequence of $x \mapsto 1 / \sqrt{x}$ being a decreasing function, and the sum being a "rightendpoint" approximation of the integral. The second inequality is a simple extension of the first, using that

$$
p_{k \mid x}^{n}(\mathbf{t}, \mathbf{x})=\frac{p_{k}^{n}(\mathbf{t}, \mathbf{x}) p_{1}^{n}\left(1-\mathbf{t}_{k}, x-\mathbf{x}_{k}\right)}{p_{1}^{n}(1, x)} .
$$

\section{APPENDIX B: TIGHTNESS}

In this section we consider the discrete time and space process

$$
\mathfrak{Z}^{\omega}(k, x ; \beta)=\mathbf{P}\left[\prod_{i=1}^{k}\left(1+\beta \omega\left(i, S_{i}\right)\right) \mathbf{1}\left\{S_{k}=x\right\}\right] .
$$

We will show that the continuous time and space processes

$$
(t, x) \mapsto z_{n}^{\omega}(t, x):=\sqrt{n} \mathfrak{Z}^{\omega}\left(n t, x \sqrt{n} ; n^{-1 / 4}\right)
$$

are tight as random elements in $C([\varepsilon, T] \times \mathbb{R})$ for any $0<\varepsilon<T<\infty$. Observe that since $\mathfrak{Z}^{\omega}$ is only defined at lattice points, it requires some interpolation to extend $z_{n}^{\omega}$ to a continuous function of space and time. The exact interpolation scheme will not really matter, but to be concrete we define it as follows: at points $(t, x) \in[0,1] \times \mathbb{R}$ such that $(t, x)$ is a corner point of the left-hand side of a rectangle in $\mathcal{R}_{n}$, define $z_{n}^{\omega}$ according to (34). Then for space-time points on the left edges 
of rectangles in $\mathcal{R}_{n}$ define $z_{n}^{\omega}$ by linear interpolation of the values on the corners that the edge connects, and finally for $(t, x)$ on the interior points of rectangles define $z_{n}^{\omega}$ by linear interpolation of the values at the four boundary corners.

The main idea for proving tightness will be to use equation (33) to get a stochastic difference equation for $\mathfrak{Z}^{\omega}$, and then transfer this to a stochastic difference equation for $z_{n}^{\omega}$ which approximates the stochastic heat equation. Standard SPDE estimates from [44], which show the regularity in space and time of the stochastic heat equation, are then shown to hold uniformly in $n$, which will prove the tightness.

REMARK. The same argument will also show the tightness for the analogously rescaled partition function

$$
e^{-n \lambda\left(\beta n^{-1 / 4}\right)} \sqrt{n} Z^{\omega}\left(n t, x \sqrt{n} ; \beta n^{-1 / 4}\right)
$$

from the exponential model

$$
Z^{\omega}(k, x ; \beta)=\mathbf{P}\left[\exp \left\{\beta \sum_{i=1}^{k} \omega\left(i, S_{i}\right)\right\} \mathbf{1}\left\{S_{k}=x\right\}\right],
$$

because it can be written in the form (33) using the field $\tilde{\omega}_{n}$ as described in Proposition 5.4 .

REMARK. Tightness of the two-parameter field (34) is sufficient to prove tightness of the four-parameter field

$$
(s, y ; t, x) \mapsto \sqrt{n} \mathfrak{Z}^{\omega}\left(n s, y \sqrt{n} ; n t, x \sqrt{n} ; n^{-1 / 4}\right),
$$

where $\mathfrak{Z}^{\omega}$ is defined by

$$
\mathfrak{Z}^{\omega}(m, y ; k, x ; \beta)=\mathbf{P}\left[\prod_{i=m+1}^{k}\left(1+\beta \omega\left(i, S_{i}\right)\right) \mathbf{1}\left\{S_{k}=x\right\} \mid S_{m}=y\right] .
$$

Indeed, tightness of (34) implies tightness of (35) in the forward $(t, x)$ variables, and tightness in the $(s, y)$ variables follows from reversibility of the random walk and the fact that the law of the environment field is invariant under a similar time reversal. More precisely, define a field $\omega_{n}$ by $\omega_{n}(i, x)=\omega(n-i, x)$. Then it is clear that

$$
\begin{aligned}
(1+ & \beta \omega(n-m, y)) \mathfrak{Z}^{\omega}(m, y ; k, x ; \beta) \\
& =(1+\beta \omega(n-k, x)) \mathfrak{Z}^{\omega_{n}}(n-k, x ; n-m, y ; \beta),
\end{aligned}
$$

hence the backward variables become the forward variables after reversing the direction of time. 
To obtain the difference equation for $\mathfrak{Z}^{\omega}$ simply condition on the step between times $k$ and $k+1$ to obtain

$$
\begin{aligned}
\mathfrak{Z}^{\omega}(k & +1, x ; \beta) \\
\quad & =\frac{1}{2}(1+\beta \omega(k+1, x))\left[\mathfrak{Z}^{\omega}(k, x+1 ; \beta)+\mathfrak{Z}^{\omega}(k, x-1 ; \beta)\right] .
\end{aligned}
$$

Throughout we let

$$
\overline{\mathfrak{Z}^{\omega}}(k, x ; \beta)=\frac{1}{2}\left[\mathfrak{Z}^{\omega}(k, x+1 ; \beta)+\mathfrak{Z}^{\omega}(k, x-1 ; \beta)\right]
$$

so that equation (36) becomes

$$
\mathfrak{Z}^{\omega}(k+1, x ; \beta)=(1+\beta \omega(k+1, x)) \overline{\mathfrak{Z}^{\omega}}(k, x ; \beta) .
$$

Observe that the first and second terms of the right-hand side are independent of each other. Subtracting $\mathfrak{Z}^{\omega}(k, x ; \beta)$ from both sides of (36) therefore yields

$$
\begin{aligned}
\mathfrak{Z}^{\omega}(k & +1, x ; \beta)-\mathfrak{Z}^{\omega}(k, x ; \beta) \\
& =\frac{1}{2} \Delta \mathfrak{Z}^{\omega}(k, x ; \beta)+\beta \omega(k+1, x) \overline{\mathfrak{Z}^{\omega}}(k, x ; \beta) .
\end{aligned}
$$

This is a discrete version of the stochastic heat equation, with initial condition $\mathfrak{Z}^{\omega}(0, x ; \beta)=\mathbf{1}\{x=0\}$. An immediate advantage of equation (37) is that it allows for an "integral form" representation of $\mathfrak{Z}^{\omega}$. In general if $Z$ is a solution to

$$
Z(k+1, x)-Z(k, x)=\frac{1}{2} \Delta Z(k, x)+f(k+1, x),
$$

then it is an easy consequence of the superposition principle that

$$
Z(k, x)=\mathrm{E}_{x}\left[Z\left(0, S_{k}\right)\right]+\sum_{i=1}^{k} \mathrm{E}_{x}\left[f\left(i, S_{k-i}\right)\right],
$$

where the expectation is over simple random walks $S$ beginning from $x$. Applying this to equation (37) yields

$$
\begin{aligned}
\mathfrak{Z}^{\omega}(k, x ; \beta) & =\mathbf{P}_{x}\left(S_{k}=0\right)+\beta \sum_{i=1}^{k} \mathrm{E}_{x}\left[\omega\left(i, S_{k-i}\right) \overline{\mathfrak{Z}^{\omega}}\left(i-1, S_{k-i} ; \beta\right)\right] \\
& =p(k, x)+\beta \sum_{i=1}^{k} \sum_{y} \omega(i, y) \overline{\mathfrak{Z}^{\omega}}(i-1, y ; \beta) p(k-i, y-x) .
\end{aligned}
$$

Now we translate this into an equation for the rescaled field (34),

$$
\begin{aligned}
z_{n}(t, x)= & p_{n}(t, x) \\
& +n^{-3 / 2} \sum_{\substack{s \in[0, t] \cap n^{-1} \mathbb{Z} \\
y \in n^{-1 / 2} \mathbb{Z}}} p_{n}(t-s, x-y) \bar{z}_{n}(s, y) \omega(s+1 / n, y),
\end{aligned}
$$


where $\bar{z}_{n}$ is the rescaled analogue of $\overline{\mathfrak{Z}^{\omega}}$, and $p_{n}(t, x)=\sqrt{n} \bar{p}_{1}(n t, x \sqrt{n})=$ $\sqrt{n} p\left(\lceil n t\rceil,[x \sqrt{n}]_{\lceil n t\rceil}\right)$ is the rescaled point-to-point transition probabilities for the random walk. Observe that these $p_{n}$ are order one in $n$, and in fact converge to $\varrho$ as $n \rightarrow \infty$. The key point, however, is the $n^{-3 / 2}$ in the second term, which is a consequence of the diffusive scaling in space and time and the intermediate scaling on the environment. By extending $\omega$ to a function that is piecewise constant on rectangles of $\mathcal{R}_{1}$, we can rewrite (38) in an integral form

$$
z_{n}(t, x)=p_{n}(t, x)+\int_{0}^{t} \int_{\mathbb{R}} p_{n}(t-s, x-y) \bar{z}_{n}(s, y) \omega(s+1 / n, y) d y d s .
$$

A key fact is that the $\omega$ terms are independent of the $\bar{z}_{n}$ terms, since the term $\bar{z}_{n}(s, \cdot)$ is a function of only the $\omega(i, \cdot)$ variables with $i \leq\lfloor n s\rfloor$. Using this fact one can derive the following a priori estimate, a proof of which can be modified from Lemma 3.1 of [4].

Lemma B.1. Suppose that $z_{n}$ satisfies (39) where the $\omega(i, x)$ are independent and identically distributed with mean zero and $M$ finite moments, where $M \geq 2$. Then there exist $C, C_{M}$ such that for any $s>0, y \in \mathbb{R}$ and $n \geq 1$,

$$
E\left[z_{n}(s, y)^{2}\right] \leq C \varrho(s, y)^{2}, \quad E\left[z_{n}(s, y)^{M}\right] \leq C_{M} \varrho(s, y)^{M} .
$$

The same bounds also extend to $\bar{z}_{n}$.

We will start the Duhamel formula (39) with the data at time $0<\varepsilon<t$,

$$
\begin{aligned}
z_{n}(t, x)= & \int p_{n}(t-\varepsilon, x-y) z_{n}(\varepsilon, y) d y \\
& \quad+\int_{\varepsilon}^{t} \int_{\mathbb{R}} p_{n}(t-s, x-y) \bar{z}_{n}(s, y) \omega(s+1 / n, y) d y d s \\
= & : A_{n, \varepsilon}(t, x)+U_{n, \varepsilon}(t, x) .
\end{aligned}
$$

We develop modulus of continuity estimates for $A_{n, \varepsilon}(t, x)$ and $U_{n, \varepsilon}(x, t)$ based on Lemma B.1. We give the details for $U_{n, \varepsilon}(x, t)$, as the analogous results for $A_{n, \varepsilon}(t, x)$ are much more straightforward. First, we use a discrete Burkholder inequality (or equivalently the Marcinkiewicz-Zygmund inequality; see, e.g., [35], page 61) which tells us that there is a constant $C_{M}<\infty$ such that

$$
\begin{aligned}
& E\left[\left|U_{n, \varepsilon}(x+\delta, t)-U_{n, \varepsilon}(x, t)\right|^{M}\right] \\
& \leq C_{M} E\left[\left(\int _ { \varepsilon } ^ { t } \int \left(p_{n}(t-s, x+\delta-y)\right.\right.\right. \\
& \left.\left.\left.\quad-p_{n}(t-s, x-y)\right)^{2} z_{n}(s, y)^{2} d y d s\right)^{M / 2}\right] .
\end{aligned}
$$


Apply Hölder's inequality with $p=M / 2, q=M /(M-2)$ to bound this by

$$
\begin{aligned}
& C_{M}^{\prime}\left(\int_{\varepsilon}^{t} \int\left(p_{n}(t-s, x+\delta-y)-p_{n}(t-s, x-y)\right)^{2 M /(M-2)} d y d s\right)^{(M-2) / 2} \\
& \quad \times E\left[\int_{\varepsilon}^{t} \int z_{n}(s, y)^{M} d y d s\right] .
\end{aligned}
$$

From Lemma B.1 we have a bound on the second term that is independent of $n$

$$
E\left[\int_{\varepsilon}^{t} \int z_{n}(s, y)^{M} d y d s\right] \leq C_{M} .
$$

Note that this bound does depend on $t$ and $\varepsilon$, but we are assuming that they are both within a compact interval that is bounded away from zero and hence there is no issue. Further, one can check that there is a $C$ also depending only on $t, \varepsilon>0$ such that

$$
\begin{aligned}
& \left(\int_{\varepsilon}^{t} \int\left(p_{n}(t-s, x+\delta-y)-p_{n}(t-s, x-y)\right)^{2 M /(M-2)} d y d s\right)^{(M-2) / 2} \\
& \quad \leq C_{M} \delta^{M / 2-1} .
\end{aligned}
$$

This is proved for the heat kernel in Walsh, and can be extended to the present discrete case by the local central limit theorem. Combining these estimates together gives the existence of a constant $C_{M}$ such that

$$
E\left[\left|U_{n, \varepsilon}(x+\delta, t)-U_{n, \varepsilon}(x, t)\right|^{M}\right] \leq C_{M} \delta^{M / 2-1} .
$$

We now produce similar estimates for $E\left[\left|U_{n, \varepsilon}(x, t+h)-U_{n, \varepsilon}(x, t)\right|^{M}\right]$. Writing out the difference we see that it splits into the sum of two terms, and using the inequality $(a+b)^{M} \leq 2^{M}\left(a^{M}+b^{M}\right)$ together with the Burkholder inequality of above, we upper bound the expectation by the sum of

$$
\begin{aligned}
C_{M} 2^{M} E\left[\left(\int _ { \varepsilon } ^ { t } \int \left(p_{n}(t+h-s, x-y)\right.\right.\right. & \\
& \left.\left.\left.\quad-p_{n}(t-s, x-y)\right)^{2} z_{n}(s, y)^{2} d y d s\right)^{M / 2}\right]
\end{aligned}
$$

and

$$
C_{M} 2^{M} E\left[\left(\int_{t}^{t+h} \int p_{n}(t+h-s, x-y)^{2} z_{n}(s, y)^{2} d y d s\right)^{M / 2}\right] .
$$

The first term (43) we bound by Hölder's inequality with $p=M / 2, q=M /(M-$ 2) to get

$$
\begin{aligned}
& E\left[\int_{\varepsilon}^{t} \int_{n} z_{n}(s, y)^{M} d y d s\right] \\
& \quad \times\left(\int_{\varepsilon}^{t} \int\left|p_{n}(t+h-s, x-y)-p_{n}(t-s, x-y)\right|^{2 M /(M-2)} d y d s\right)^{(M-2) / 2} .
\end{aligned}
$$


The expectation term is bounded above by (41). For $M>6$, the second term is uniformly bounded in $n$, again by using the local limit theorem to perform the estimate for the heat kernel rather than the random walk kernel. The upper bound is $C_{M} h^{M / 4-2}$.

We also use Hölder on (44) to get an upper bound of

$$
\begin{aligned}
& E\left[\int_{t}^{t+h} \int z_{n}(s, y)^{M} d y d s\right] \\
& \quad \times\left(\int_{t}^{t+h} \int p_{n}(t+h-s, x-y)^{2 M /(M-2)} d y d s\right)^{(M-2) / 2} .
\end{aligned}
$$

Again one uses the a priori bound of Lemma B.1 and heat kernel estimates to get that this is less than $C_{M} h^{M / 4-2}$. Therefore we have the existence of a constant $C_{M}$ such that

$$
E\left[\left|U_{n, \varepsilon}(x, t+h)-U_{n, \varepsilon}(x, t)\right|^{M}\right] \leq C_{M} h^{M / 4-2} .
$$

Combining (42) with (45) and the equation (40) we have the following:

LEMMA B.2. For each even $M>6$, and each $\varepsilon>0$, there is a $C_{M}<\infty$ such that for $t, t+h \geq \varepsilon$, and all $n \geq 0$,

$$
E\left[\left|z_{n}(x+\delta, t+h)-z_{n}(x, t)\right|^{M}\right]^{1 / M} \leq C_{M}(|\delta|+|h|)^{1 / 4-2 / M} .
$$

Now we use the inequality of Garsia [23] that

$$
|f(x)-f(y)| \leq 8 \int_{0}^{|x-y|} \Psi^{-1}\left(B / u^{2 d}\right) d p(u)
$$

for all functions $f$ continuous in a unit cube $I \subset \mathbf{R}^{d}$ that satisfy the inequality

$$
\int_{I} \int_{I} \Psi(f(x)-f(y)) / p\left(d^{-1 / 2}|x-y|\right) d x d y \leq B
$$

where (i) $\Psi$ is nonconstant positive even convex function with $\lim _{x \rightarrow \infty} \Psi(x)=$ $\infty$, and (ii) $p$ is a positive continuous even function increasing on $(0, \infty)$ that satisfies the condition $\lim _{u \rightarrow 0} p(u)=0$.

We are working in $d=2$ (space + time). Choosing $\Psi(x)=x^{M}, M>6$ and $p(x)=x^{\gamma / M}$, we have from Lemma B.2.

$$
E\left[\int_{t, s \in[\varepsilon, T], x, y \in \mathbb{R}} \Psi\left(\frac{\left|z_{n}(x, t)-z_{n}(y, s)\right|}{p\left(2^{-1 / 2} \sqrt{(t-s)^{2}+(x-y)^{2}}\right)}\right)\right] \leq C_{M} .
$$

Since $\int_{0}^{h} \Psi^{-1}\left(B / u^{2 d}\right) d p(u)=C_{M, \gamma} B^{1 / M} h^{(\gamma-4) / M}$ with a finite $C_{M, \gamma}$ for $\gamma>4$, we conclude that if $\mathcal{H}_{[\varepsilon, T] \times \mathbb{R}}(\alpha, K)$ denotes the set of functions $z(t, x)$ on $[\varepsilon, T] \times$ $\mathbb{R}$ with $|z(t, x)-z(s, y)| \leq K\left|(t-s)^{2}+(x-y)^{2}\right|^{\alpha / 2}$, then we have the following: 
LEMMA B.3. If $P_{n}$ denotes the distribution of $z_{n}(t, x)$, then for any $\varepsilon>0$ and $\alpha<1 / 4$,

$$
\limsup _{K \rightarrow \infty} \limsup _{n \rightarrow \infty} P_{n}\left(C([\varepsilon, T] \times \mathbb{R}) \backslash \mathcal{H}_{[\varepsilon, T] \times \mathbb{R}}(\alpha, K)\right)=0 .
$$

In particular, since $\mathcal{H}_{[\varepsilon, T] \times \mathbb{R}}(\alpha, K)$ are compact sets of $C([\varepsilon, T] \times \mathbb{R})$, the $P_{n}$ are tight.

REMARK. If we had been more careful we could improve the modulus of continuity to Hölder $1 / 2-$ in space, but for tightness we do not need an optimal result.

Acknowledgments. The authors would like to thank Ivan Corwin and several anonymous referees for helpful comments and literature references that led to a much improved presentation.

\section{REFERENCES}

[1] Abramowitz, M. and Stegun, I. A. (1964). Handbook of Mathematical Functions with Formulas, Graphs, and Mathematical Tables. National Bureau of Standards Applied Mathematics Series 55. U.S. Government Printing Office, Washington, DC. MR0167642

[2] Alberts, T., Khanin, K. and QuAstel, J. (2014). The continuum directed random polymer. J. Stat. Phys. 154 305-326. MR3162542

[3] Alberts, T., Khanin, K. and QuAstel, J. (2010). Intermediate disorder regime for directed polymers in dimension 1+1. Phys. Rev. Lett. 105090603.

[4] Amir, G., Corwin, I. and QuAstel, J. (2011). Probability distribution of the free energy of the continuum directed random polymer in $1+1$ dimensions. Comm. Pure Appl. Math. 64 466-537. MR2796514

[5] Auffinger, A. and Damron, M. (2011). A simplified proof of the relation between scaling exponents in first-passage percolation. Available at arXiv:1109.0523v1 [math.PR].

[6] Bertini, L. and Giacomin, G. (1997). Stochastic Burgers and KPZ equations from particle systems. Comm. Math. Phys. 183 571-607. MR1462228

[7] Billingsley, P. (1968). Convergence of Probability Measures. Wiley, New York. MR0233396

[8] Biroli, G., Bouchaud, J. P. and Potters, M. (2007). On the top eigenvalue of heavy-tailed random matrices. Europhys. Lett. EPL 78 Art. 10001, 5. MR2371333

[9] Biroli, G., Bouchaud, J.-P. and Potters, M. (2007). Extreme value problems in random matrix theory and other disordered systems. J. Stat. Mech. Theory Exp. 7 P07019, 15 pp. (electronic). MR2335697

[10] Bolthausen, E. (1989). A note on the diffusion of directed polymers in a random environment. Comm. Math. Phys. 123 529-534. MR1006293

[11] Calabrese, P. and Doussal, P. L. (2011). Exact solution for the Kardar-Parisi-Zhang equation with flat initial conditions. Phys. Rev. Lett. 106250603.

[12] Calabrese, P., Le Doussal, P. and Rosso, A. (2010). Free-energy distribution of the directed polymer at high temperature. Europhys. Lett. EPL 9020002.

[13] Carmona, P. and Hu, Y. (2002). On the partition function of a directed polymer in a Gaussian random environment. Probab. Theory Related Fields 124 431-457. MR1939654

[14] Chatterjee, S. (2013). The universal relation between scaling exponents in first-passage percolation. Ann. of Math. (2) 177 663-697. MR3010809 
[15] Comets, F., Shiga, T. and Yoshida, N. (2003). Directed polymers in a random environment: Path localization and strong disorder. Bernoulli 9 705-723. MR1996276

[16] Comets, F., Shiga, T. and Yoshida, N. (2004). Probabilistic analysis of directed polymers in a random environment: A review. In Stochastic Analysis on Large Scale Interacting Systems. Adv. Stud. Pure Math. 39 115-142. Math. Soc. Japan, Tokyo. MR2073332

[17] Comets, F. and VARGas, V. (2006). Majorizing multiplicative cascades for directed polymers in random media. ALEA Lat. Am. J. Probab. Math. Stat. 2 267-277. MR2249671

[18] Comets, F. and YoshidA, N. (2005). Brownian directed polymers in random environment. Comm. Math. Phys. 254 257-287. MR2117626

[19] Comets, F. and Yoshida, N. (2006). Directed polymers in random environment are diffusive at weak disorder. Ann. Probab. 34 1746-1770. MR2271480

[20] Durrett, R. (2010). Probability: Theory and Examples, 4th ed. Cambridge Univ. Press, Cambridge. MR2722836

[21] Dynkin, E. B. and Mandelbaum, A. (1983). Symmetric statistics, Poisson point processes, and multiple Wiener integrals. Ann. Statist. 11 739-745. MR0707925

[22] FENG, Z. S. (2012). Diffusivity of rescaled random polymer in random environment in dimensions 1 and 2. Available at arXiv:1201.6215v1 [math.PR].

[23] GARSIA, A. M. (1972). Continuity properties of Gaussian processes with multidimensional time parameter. In Proceedings of the Sixth Berkeley Symposium on Mathematical Statistics and Probability (Univ. California, Berkeley, CA, 1970/1971), Vol. II: Probability Theory 369-374. Univ. California Press, Berkeley, CA. MR0410880

[24] GINE, E. (1997). Decoupling and limit theorems for $U$-statistics and $U$-processes. In Lectures on Probability Theory and Statistics (Saint-Flour, 1996). Lecture Notes in Math. 1665 1-35. Springer, Berlin. MR1490043

[25] Halpin-Healy, T. and Zhang, Y.-C. (1995). Kinetic roughening phenomena, stochastic growth, directed polymers and all that aspects of multidisciplinary statistical mechanics. Phys. Rep. 254 215-414.

[26] Huse, D. A. and Henley, C. L. (1985). Pinning and roughening of domain walls in ising systems due to random impurities. Phys. Rev. Lett. 54 2708-2711.

[27] Imbrie, J. Z. and SPENCER, T. (1988). Diffusion of directed polymers in a random environment. J. Stat. Phys. 52 609-626. MR0968950

[28] Janson, S. (1997). Gaussian Hilbert Spaces. Cambridge Tracts in Mathematics 129. Cambridge Univ. Press, Cambridge. MR1474726

[29] Kardar, M., Parisi, G. and Zhang, Y.-C. (1986). Dynamic scaling of growing interfaces. Phys. Rev. Lett. 56 889-892.

[30] Lacoin, H. (2010). New bounds for the free energy of directed polymers in dimension $1+1$ and $1+$ 2. Comm. Math. Phys. 294 471-503. MR2579463

[31] Mejane, O. (2004). Upper bound of a volume exponent for directed polymers in a random environment. Ann. Inst. Henri Poincaré Probab. Stat. 40 299-308. MR2060455

[32] Nolan, D. and Pollard, D. (1987). U-processes: Rates of convergence. Ann. Statist. 15 780-799. MR0888439

[33] Nolan, D. and Pollard, D. (1988). Functional limit theorems for $U$-processes. Ann. Probab. 16 1291-1298. MR0942769

[34] Petermann, M. (2000). Superdiffusivity of directed polymers in random environment. Ph.D. thesis, Univ. Zürich.

[35] Petrov, V. V. (1975). Sums of Independent Random Variables. Springer, New York. MR0388499

[36] PrÄhofer, M. and Spohn, H. (2002). Scale invariance of the PNG droplet and the Airy process. J. Stat. Phys. 108 1071-1106. MR1933446

[37] Sasamoto, T. and SPOHN, H. (2010). Universality of the one-dimensional KPZ equation. Available at arXiv:1002.1883v2 [cond-mat.stat-mech]. 
[38] Sasamoto, T. and Spohn, H. (2010). Exact height distributions for the KPZ equation with narrow wedge initial condition. Nuclear Phys. B 834 523-542. MR2628936

[39] SEPPÄLÄINEN, T. (2012). Scaling for a one-dimensional directed polymer with boundary conditions. Ann. Probab. 40 19-73. MR2917766

[40] Tracy, C. A. and Widom, H. (1994). Level-spacing distributions and the Airy kernel. Comm. Math. Phys. 159 151-174. MR1257246

[41] Tracy, C. A. and Widom, H. (2008). A Fredholm determinant representation in ASEP. J. Stat. Phys. 132 291-300. MR2415104

[42] TRACY, C. A. and WidOM, H. (2008). Integral formulas for the asymmetric simple exclusion process. Comm. Math. Phys. 279 815-844. MR2386729

[43] Tracy, C. A. and Widom, H. (2009). Asymptotics in ASEP with step initial condition. Comm. Math. Phys. 290 129-154. MR2520510

[44] Walsh, J. B. (1986). An introduction to stochastic partial differential equations. In École D'été de Probabilités de Saint-Flour, XIV-1984. Lecture Notes in Math. 1180 265-439. Springer, Berlin. MR0876085

[45] WÜthrich, M. V. (1998). Fluctuation results for Brownian motion in a Poissonian potential. Ann. Inst. Henri Poincaré Probab. Stat. 34 279-308. MR1625875

[46] WÜthrich, M. V. (1998). Superdiffusive behavior of two-dimensional Brownian motion in a Poissonian potential. Ann. Probab. 26 1000-1015. MR1634412

T. Alberts

DEPARTMENT OF MATHEMATiCS

CALifornia Institute of TeChNOLOGY

MC 253-37

1200 E CALIFORNIA BLVD.

PASADENA, CALIFORNIA 91125

USA
K. KHANIN

J. QUASTEL

DEPARTMENT OF MATHEMATICS

UNIVERSITY OF TORONTO

Room 6290, 40 St. George St.

TORONTO, ONTARIO M5S 2E4

CANADA 